\title{
1 Botrytis cinerea BcPTP1 is a late infection phase, cysteine rich protein
}

3 Wenjun $\mathrm{Zhu}^{1}{ }^{*}$, Mengxue $\mathrm{Yu}^{1}$, Ran $\mathrm{Xu}^{1}$, Kai $\mathrm{Bi}^{1}$, Chao Xiong ${ }^{1}$, Zhiguo Liu ${ }^{1}$, Amir

4 Sharon $^{2}$, Daohong Jiang ${ }^{3}$, Mingde $\mathrm{Wu}^{3}$, Qiongnan $\mathrm{Gu}^{4}$, Ling Gong ${ }^{5}$, Weidong Chen ${ }^{6}$,

5 Wei Wei ${ }^{6 *}$

6

7 1. School of Life Science and Technology, Wuhan Polytechnic University, Wuhan

8 430023, Hubei Province, China.

9

10

2. School of Plant Sciences and Food Security, Faculty of Life Sciences, Tel Aviv University, Tel Aviv 69978, Israel.

3. State Key Laboratory of Agricultural Microbiology, Huazhong Agricultural University, Wuhan 430070, Hubei Province, China.

4. Institute of Plant Protection and Soil Fertilizer, Hubei Academy of Agricultural Sciences/Key Laboratory of Integrated Pest Management on Crops in Central China, Ministry of Agriculture and Rural Affairs/Hubei Key Laboratory of Crop Diseases, Insect Pests and Weeds control, Wuhan 430064, Hubei Province, China.

5. Pharmacy Faculty, Hubei University of Chinese Medicine, Wuhan 430065, Hubei Province, China.

6. Department of Plant Pathology, Washington State University, United States Department of Agriculture-Agricultural Research Service, Washington State University, Pullman 99164, USA.

*Corresponding authors.

E-mail addresses:

Wenjun Zhu: zhuwenjun2017@whpu.edu.cn

Wei Wei: wei.wei2@wsu.edu 


\section{$34 \quad$ Abstract}

Botrytis cinerea is a broad-host-range necrotrophic phytopathogen responsible for serious crops diseases. To facilitate infection, $B$. cinerea secretes a large number of effectors that induce plant cell death. In screening secretome data of $B$. cinerea during infection stage, we identified a phytotoxic protein (BcPTP1) that can also induce immune resistance in plants. BcPTP1 is a small (90 aa), cysteine rich protein without any known domains. Transiently expression of BcPTP1 in leaves caused chlorosis that intensifies with time and eventually lead to cell death. Point mutations in eight of the 10 cysteine residues of BcPTP1 abolished the toxic effect, however residual toxic activity remained after heating the peptide, suggesting contribution of unknown epitopes to protein phytotoxic effect. The transcript level of the bcptpl gene was low during the first $36 \mathrm{~h}$ after inoculation and increased sharply upon transition to the late infection stage, suggesting a role of BcPTP1 in lesion spreading. While statistically insignificant, deletion of the bcptpl gene led to slightly smaller lesions on bean leaves. Further analyses indicated that BcPTP1 is internalized into plant cells after secreting into the apoplast and its phytotoxic effect is negatively regulated by the receptor-like kinases BAK1 and SOBIR1. Collectively, our findings show that BcPTP1 is a virulence factor that toxifies the host cells and facilitates lesion spreading during the late infection stage.

Keywords: Botrytis cinerea, effector, cell death, phytotoxic protein, immune resistance, receptor-like kinases

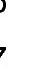

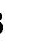

(1)

1

3

4


72

73

74

75

76

77

78

79

80

81

82

83

84

85

86

87

88

89

90

91

92

93

94

95

96

97

98

99

100

101

102

103

104

105

106

107

108

109

\section{Introduction}

During the evolutionary arms race with phytopathogens, plants have developed a sophisticated defense system (Jones and Dangl, 2006). The first defense layer is pathogen-associated molecular patterns (PAMPs)-triggered immunity (PTI), in which host receptors recognize molecules or domains that are conserved in certain groups of pathogens and induce an effective resistance response (Albert et al., 2020). In fungi and oomycetes, the best studied PAMPs include glucans (Fliegmann et al., 2004), xylanase EIX (Rotblat et al., 2002), chitin (Shinya et al., 2015), endopolygalacturonases (Zhang et al., 2014b), Pep13 (Brunner et al., 2002), Cerato-platanin proteins (Yang et al., 2018) and INF1 (Kanneganti et al., 2006). In addition, several PAMPs that induce plant necrosis or PTI across different classes of microbes have also been well characterized, such as glycoside hydrolase 12 proteins (GH12) in the fungal pathogen Verticillium dahliae and Botrytis cinerea (Gui et al., 2017; Zhu et al., 2017) and the oomycete pathogen Phytophthora sojae (Ma et al., 2015; Ma et al., 2017; Wang et al., 2018), Ave1 protein in multiple plant pathogenic fungi and bacteria (Thomma et al., 2011; de Jonge et al., 2012), and the classical necrosis and ethylene-inducing peptide 1-like proteins (NLPs) in multiple prokaryotic and eukaryotic microbial pathogens (Oome et al., 2014; Albert et al., 2015).

To overcome the basal plant immunity and infect hosts effectively, biotrophic and hemibiotrophic pathogens secrete diverse effectors to the interface area between fungal hyphae and host or into plant cells (Stergiopoulos and de Wit, 2009; Lo Presti et al., 2015; Kim et al., 2016). To cope with pathogen effectors, plants have co-evolved a second line of defense, also called effector-triggered immunity (ETI), which is mediated by resistance $(\mathrm{R})$ proteins and is often associated with the local plant cells hypersensitive response (HR) at the infection site (Cui et al., 2015). HR is efficient in restricting biotrophic and hemibiotrophic pathogens (Jones and Dangl, 2006; van Ooijen et al., 2007; Cui et al., 2015), but is ineffective against necrotrophic pathogens, which can turn it against the host to facilitate infection (Lorang et al., 2012; Gao et al., 2015).

Compared to the large number and well characterized effectors in biotrophic and hemibiotrophic fungal pathogens, relatively fewer effectors have been studied in necrotrophic fungal pathogens, especially the broad-host-range necrotrophic fungal pathogens. Botrytis cinerea is a typical broad-host-range necrotrophic fungal phytopathogen, causing gray mold and rot diseases in hundreds of plant species including many agriculturally important crops, and leading to enormous economic losses each year (Dean et al., 2012; Veloso and van Kan, 2018). It was shown that the infection process of $B$. cinerea on host plant includes three typical stages: an early stage characterized by local necrosis lesions formation, an intermediate stage during 
which a variety of sophisticated interactions between plant-pathogen occur and determine the outcome of $B$. cinerea infections, and the late stage of fast-spreading lesions (Eizner et al., 2017). Since B. cinerea infects and colonizes by killing plant cells, it secretes diverse effector proteins to manipulate the host defenses and/or to induce death for facilitating infection on host plants (Heard et al., 2015; Frías et al., 2016; Zhu et al., 2017; Denton-Giles et al., 2020; Frías et al., 2011; Shao et al., 2021). These secreted effector proteins play important roles during Botrytis-plant interactions. However, compared with biotrophic and hemibiotrophic pathogens effectors, the underlying biochemical activities and molecular mechanisms of the necrotrophic secreted proteins remain incompletely understood.

Aiming to characterize potential effectors of $B$. cinerea, we identified a secreted protein with a phytotoxic activity that was named BcPTP1 (phytotoxic protein). Similar to most other cell death inducing proteins (CDIPs, Li et al., 2020; Shao et al., 2021), BcPTP1 functions in apoplastic space of plant cell and induces a plant defense response. However, unlike the vast majority of CDIPs, BcPTP1 leads to development of gradual chlorosis rather than instant cell death and the bcptpl gene is expressed late during infection and affects lesion development, namely it is a late-stage virulence factor.

\section{Results}

\section{BcPTP1 is a secreted protein with phytotoxic activity}

The effect of putative effector proteins was tested by Agrobacterium tumefaciens-mediated transient expression (agroinfiltration) of the candidate proteins fused with GFP tag at the C-terminus in $N$. benthamiana leaves. We found that the protein BcPTP1 (BCIN_05g03680)-GFP containing a potential N-terminal signal peptide caused chlorosis in $N$. benthamiana leaves that developed within 10 days after agroinfiltration, eventually leading to cell death within 15 days after agroinfiltration (Fig. 1). Transient expression of BcPTP $1^{\triangle \mathrm{SP}}$-GFP without the secretion signal peptide did not trigger leaf chlorosis, indicating that BcPTP1 may function in the leaf apoplastic space. This phenotype differs from the induction of necrotic cell death that is common to previously characterized apoplastic CDIPs (Li et al., 2020; Shao et al., 2021), and suggests that BcPTP1 has a phytotoxic activity rather than a necrotic cell death inducing activity.

Two methods were used to verify whether BcPTP1 is a secreted protein. First, the BcPTP1 signal peptide (initial $\mathrm{N}$ terminus 19 amino acids) was used to replace the $\mathrm{N}$-terminal signal peptide of BcXYG1, the strong death-inducing secreted protein of $B$. cinerea which functions in the apoplastic space (Zhu et al., 2017), to produce $\mathrm{SP}^{(\mathrm{BcPTP} 1)}-\mathrm{BcXYG1}{ }^{\triangle \mathrm{SP}}-\mathrm{HA}$. The BcXYG1-HA with its native signal peptide was used 
as a positive control, and the $\mathrm{BcXYG1}{ }^{\triangle \mathrm{SP}}$-HA, GFP-HA, $\mathrm{SP}^{(\mathrm{BcPTP} 1)}-\mathrm{GFP}-\mathrm{HA}$ and empty vector were used as negative controls. Within five days after agroinfiltration, both of BcXYG1-HA and $\mathrm{SP}^{(\mathrm{BcPTP} 1)}-\mathrm{BcXYG} 1^{\triangle \mathrm{SP}}-\mathrm{HA}$ fusion proteins, which are secreted from the plant cells to the extracellular space, triggered typical plant cell death, whereas BcXYG1 ${ }^{\Delta \mathrm{SP}}$-HA, which lacks the signal peptide and therefore remains inside the plant cell, failed to induce cell death (Fig. 2A). In the second method, we fused the signal peptide of BcPTP1 to GFP to form $\mathrm{SP}^{(\mathrm{BcPTP} 1)}-\mathrm{GFP}$. Likewise, we fused the signal peptide of BcXYG1 to GFP to form $\mathrm{SP}^{(\mathrm{BcXYG1})}-\mathrm{GFP}$ for comparison. The constructs were used to transform the wild type $B$. cinerea strain to generate overexpression strains of these fusion proteins. All the indicated proteins were successfully expressed in the hyphae of each strain as shown in western blot analysis (Fig. 2C). Then, all examined strains were cultured in potato dextrose broth (PDB) for 3 days, the culture filtrates were collected, purified and presence of the fused proteins was checked by western blot analysis. The results confirmed the accumulation of GFP protein in the culture medium of $\mathrm{SP}^{(\mathrm{BcPTP} 1)}-\mathrm{GFP}$ and $\mathrm{SP}^{(\mathrm{BcXYG1})}$-GFP overexpression strains, but not the GFP overexpression or wild type (WT) strain (Fig. 2D). Collectively, these analyses confirmed the secretion function of the BcPTP1 signal peptide.

\section{BcPTP1 is internalized into plant cell}

The above results showed that BcPTP1 is a secreted protein and its phytotoxic activity depends on localization in the leaf apoplastic space (Fig. 1; Fig. 2). Thus, we postulated that BcPTP1 remains the in apoplastic space after secretion by the fungus. To verify this hypothesis, we analyzed the subcellular localization of BcPTP1-GFP fusion protein following agroinfiltration. Unexpectedly, we found that the BcPTP1-GFP fusion protein was mainly localized at the cytoplasmic vesicles and in the periphery of plant cell plasma membrane, whereas the BcPTP $1^{\triangle \mathrm{SP}}{ }_{-\mathrm{GFP}}$ and GFP were mainly distributed in the nuclei and cytoplasm, and the $\mathrm{SP}^{\mathrm{BCPTP} 1}-\mathrm{GFP}$ was concentrated in the apoplastic space (Fig. 3). The unexpected localization of BcPTP1 indicates that besides phytotoxic activity in apoplast, BcPTP1 can be also internalized into plant cells for other unknown functions after the initial secretion to apoplastic space.

\section{BcPTP1 is toxic to dicot but not monocot plants}

To determine whether BcPTP1 affects plants other than N. benthamiana and to avoid the A. tumefaciens incompatibility issues on plants, we produced and purified the BcPTP1 protein from Escherichia coli (Supplementary Fig. S1). Infiltration assay with different concentrations of the purified protein demonstrated that $25 \mu \mathrm{g} / \mathrm{ml}$ of $\mathrm{BcPTP1}$ recombinant protein was sufficient to trigger leaf chlorosis in $N$. 
187

188

189

190

191

192

193

194

195

196

197

198

199

200

201

202

203

204

205

206

207

208

209

210

211

212

213

214

215

216

217

218

219

220

221

222

223

224

225

benthamiana (Fig. 4A). Cell death developed following treatment of the leaves with higher protein titers $(50-100 \mu \mathrm{g} / \mathrm{ml})$. In addition, BcPTP1 also induced cell death in tomato and $A$. thaliana leaves, but not in the monocot maize even at high protein concentrations (Fig. 4B). These results indicated that BcPTP1 is toxic to multiple dicot plants, but not to monocot cereal.

\section{Cysteine residues in BcPTP1 are important for protein toxicity}

bcptpl is a single-copy gene in B. cinerea, encoding 90 amino acids with 10 cysteine residues, out of which eight residues are highly conserved (Supplementary Fig. S2). Bioinformatics analysis demonstrated that the first 20 N-terminal amino acids encode a signal peptide. No other known protein domains or possible functions were predicted using SMART MODE analysis (http://smart.embl-heidelberg.de/smart/change_mode.pl), and also no nuclear localization signal or chloroplast transit peptide were found in protein sequence. BLAST searches against the NCBI database with the BcPTP1 sequence as query showed that homologs of BcPTP1 with high similarity are only present in a small number of fungal genera, most of which are plant pathogens, including Botryotinia, Sclerotinia, Alternaria, Bipolaris, Fusarium, Colletotrichum, Botrytis, and saprotrophic Aspergillus species. Significantly, no homologs were found in any of the biotrophic plant pathogens or in human pathogenic fungi. Multiple sequence alignment and phylogenetic analysis of BcPTP1 and its homologues showed significant sequence similarity (Supplementary Fig. S2A and S2B). 3D structure prediction of BcPTP1 using I-TASSER showed that BcPTP1 contains two $\alpha$-helices on each side of the protein structure and two internal $\beta$-strands (Supplementary Fig. S2C).

Many effectors are small secreted cysteine-rich proteins (SSCP). The cysteine residues contribute to the formation of disulfide bonds (Sevier and Kaiser, 2002; Marianayagam et al., 2004), which are essential for the structure and function of these proteins (Stergiopoulos and de Wit, 2009). To examine whether phytotoxicity of BcPTP1 depends on its cysteine residues, we replaced each of the 10 cysteine residues with alanine individually using site-directed mutagenesis. A. tumefaciens infiltration assay of $N$. benthamiana leaves demonstrated that mutations of $\mathrm{C} 26 \mathrm{~A}$ or $\mathrm{C} 34 \mathrm{~A}$ individually could induce more severe phenotype than the native BcPTP1 protein. The activity of the C67A mutant decreased compared to the native protein, whereas mutations in any of the seven remaining cysteine residues completely abolished phytotoxicity (Fig. 5). Additionally, boiling the BcPTP1 protein at $100 \square$ for $30 \mathrm{~min}$ reduced, but did not completely abolished phytotoxicity (Fig. 6). These results suggest that certain epitopes, which are affected by specific cysteine residues, mediate the phytotoxic activity of the BcPTP1 protein. 
bcptp1 is highly expressed at late infection stage but is not essential for pathogenicity

To analyze the biological roles of BcPTP1 during $B$. cinerea infection, we measured the expression levels of the bcptpl gene during infection. The transcript levels of bcptpl were low at early infection stage and then increased sharply about 48 hpi with a peak at $60 \mathrm{hpi}$, about 35 -fold higher than at 0 hpi (Fig. 7). When B. cinerea was cultured on solid Gamborg's B5 medium, the transcript level of bcptp1 was increased about 12-fold at 36 hpi compared to earlier time points, and then remained stable throughout the culture period (Fig. 7).

To further investigate possible role of BcPTP1 in B. cinerea pathogenicity, we generated bcptpl gene deletion and overexpression strains (Supplementary Fig. S3). All the deletion and overexpression strains had normal colony morphology, conidia production and growth rate on potato dextrose agar (PDA) (Supplementary Fig. S4). In addition, we did not observe obvious changes in sensitivity of the transgenic strains to various types of stresses, including $1 \mathrm{M} \mathrm{NaCl}, 1 \mathrm{M}$ sorbitol, 0.02\% SDS, $20 \mathrm{mM}$ $\mathrm{H}_{2} \mathrm{O}_{2}, 0.3 \mathrm{mg} / \mathrm{ml}$ Calcofluor White and $0.5 \mathrm{mg} / \mathrm{ml}$ Congo Red (Supplementary Fig. S5).

Pathogenicity study showed that the bcptpl deletion strains caused slightly smaller disease lesion on bean leaves than the wild type strain, although the differences were statistically insignificant (Supplementary Fig. S6). Likewise, the bcptpl over expression strains did not show obvious difference in lesion size (Supplementary Fig. S6). The results indicated that deletion or overexpression of bcptpl does not significantly affect the final outcome of $B$. cinerea infection.

BcPTP1 triggers immune response and induces resistance against $B$. cinerea in $N$. benthamiana

High proportion of the analyzed CDIPs are recognized by the plant immune system and activate a defense response. To analyze whether BcPTP1 can induce plant resistance to $B$. cinerea, $N$. benthamiana leaves were infiltrated with $10 \mu \mathrm{g} / \mathrm{ml}$ of purified BcPTP1 or GFP proteins, $48 \mathrm{~h}$ later, the infiltrated leaves were inoculated with $B$. cinerea mycelia plugs, the plants were incubated for an additional $48 \mathrm{~h}$ in a moist chamber and then symptoms were recorded. The results showed that the infection on tobacco leaves pre-treated with BcPTP1 protein was significantly reduced compared to leaves pre-treated with GFP protein (Fig. 8A), indicating that along with phytotoxicity, BcPTP1 can also trigger plant resistance.

To verify whether the enhanced resistance of tobacco caused by BcPTP1 is 
265

266

267

268

269

270

271

272

273

274

275

276

277

278

279

280

281

282

283

284

285

286

287

288

289

290

291

292

293

294

295

296

297

298

299

300

301

302

Jasmonic acid (JA) signal pathway genes $N b P R 4$ and $N b L O X$, ethylene signal pathway gene NbERF1, HR-related genes HIN1, and PTI-related genes NbWRKY7 and NbPTI5 using RT-qPCR, as previously described (Nie et al., 2019). In all cases we observed a drastic increase in gene expression after infiltration of the leaves with $10 \mu \mathrm{g} / \mathrm{ml} \mathrm{BcPTP1}$ (Fig. 8B).

\section{BAK1 and SOBIR1 negatively regulate the death-inducing activity of BcPTP1 in} N. benthamiana

Since BcPTP1 is targeted to the apoplast space of $N$. benthamiana tissue, it is possible that similar to other CDIPs, BcPTP1 interacts with plant membrane receptor-like proteins (RLPs) to transmit the immunity signals via the RLP-SOBIR1-BAK1 complex (Liebrand et al., 2013; Zhang et al., 2014b; Albert et al., 2015; Ma et al., 2015; Postma et al., 2016; Gui et al., 2017; Zhu et al., 2017). To test this possibility, we generated NbBAKI- and NbSOBIRI-silenced N. benthamiana plants using virus-induced gene silencing (VIGS). The gene-silenced plants were agroinfiltrated with BcPTP1 expression construct. Unexpectedly, BcPTP1 induced a much more severe phytotoxicity in the BAKI- or SOBIRI-silenced plants than in wild type tobacco plants (Fig. 9). The result indicated that the death-inducing signal of BcPTP1 is mediated through an unknown signal transduction pathway, and is negatively regulated by BAK1 and SOBIR1.

\section{Discussion}

The search for $B$. cinerea cell death-inducing effectors has yielded an increasing number of candidates DCIPs, most of which are associated with production of local necrosis during the early infection stage (Li et al., 2020; Shao et al., 2021). In search of factors that facilitate disease progression in late infection stage (lesion spreading), we identified BcPTP1, a SSCP with phytotoxic activity, which is expressed at the late infection stage. As such, BcPTP1 fulfills the definition of a late-stage necrotrophic effector.

The late in planta induction of bcptpl (Fig. 7) supports a potential role of BcPTP1 in the pathogenicity of B. cinerea at the late infection stage, probably as a factor that facilitates lesion expansion. Since the BcPTP1 is recognized by the plant immune system (Fig. 8), the late expression of the bcptpl gene might also prevent early perception and induction of a plant defense response. Despite the phytoxicity and induction in planta, deletion of bcptpl gene had only minor effect on fungal virulence (Supplementary Fig. S6). Such minor, or even lack of a visible change in virulence is common to many fungal effectors, and probably reflects presence of similar effectors with a redundant function or a quantitative effect that adds to the 
303

304

305

306

307

308

309

310

311

312

313

314

315

316

317

318

319

320

321

322

323

324

325

326

327

328

329

330

331

332

333

334

335

336

337

338

339

340

341

overall virulence arsenal of the fungus.

Compared to other well studied CDIPs, which possess strong activity and trigger death of tobacco leaves within less than 5 days (Ma et al., 2015; Zhang et al., 2017; Zhu et al., 2017; Yang et al., 2018; Bi et al., 2021; Yang et al., 2021; Yin et al., 2021), treatment of leaves with purified BcPTP1 protein or transient expression of using agroinfiltration, both induced chlorosis rather than instant cell death (Fig. 1; Fig. 4A). Hence, the BcPTP1 protein probably does not cause direct damage to the plant cell but more likely, it affects some unknown pathways that eventually lead to cell death, such as accumulation of toxic metabolites or inhibition of photosynthesis.

Homologues of BcPTP1 were found in several additional fungal species, mostly necrotrophic and hemibiotrophic plant pathogens (Supplementary Fig. S2). Significantly, no homologues are found in biotrophic plant pathogens or in human pathogens, which hint to a specific role of the protein in promoting necrotrophic infection. Homologues were also found in saprophytic Aspergillus species that colonize and grow on dead plant residues. While these saprotrophic fungi probably do not need the death-inducing activity of the BcPTP1 homologs during their entire life cycle, it is possible that they benefit from an unknown activity of these proteins, or they might have a yet unidentified plant associated phase.

Many effectors contain multiple cysteine residues, which form disulphide bonds (Sevier and Kaiser, 2002; Lu and Edwards, 2016). These residues play significant roles in protein folding, structural stability and protection of such proteins against degradation in harsh acidic and protease-rich environment when they are delivered into plant apoplast during infection (Rep, 2005). BcPTP1 contains 10 cysteine residues that are predicted to form potential disulphide bonds. Mutation of seven out of the 10 cysteine residues in BcPTP1 completely abolished, and an additional one greatly reduced, the phytotoxic activity, whereas mutation of two other residues enhanced the activity (Fig. 5A). It is possible that the majority of the cysteine residues are involved in forming the proper tertiary structure of BcPTP1, thus changing these cysteine residues abolished or decreased the death-inducing activity. The other two residues might be part of epitopes that contribute to the cell death-inducing activity and the mutations might lead to exposure of the immunogenic epitopes, resulting in enhanced activity. Similar study recently revealed that the secreted apoplastic protein PC2 from the potato late blight pathogen Phytophthora infestans was cleavage by plant apoplastic proteases, which is the essential process for PC2 to release the immunogenic peptides, thus to activate plant defense responses (Wang et al., 2021a). Indeed, several studies demonstrated that heat denaturation or structural mutation of some secreted proteins did not abolished the necrosis-inducing activity (Zhang et al., 2014a; Zhang et al., 2014b), indicating that the potential epitopes are sufficient for the plant cell death-inducing activity. Similarly, our study also showed that 
342 heat-denatured BcPTP1 still retained a weak cytotoxic activity (Fig. 6). Accordingly,

343 it is possible that in addition to tertiary structure, the unknown immunogenic peptide

344 also contributes to the cell death inducing activity of BcPTP1.

Our study showed that BcPTP1 activates an immune response in N. benthamiana as measured by infection assay and induced expression of defense genes (Fig. 8). Thus, the BcPTP1 may also function as a potential elicitor. Indeed, our results demonstrated that BcPTP1 is secreted extracellularly (Fig. 1; Fig. 2) and may interact with plants receptor-like kinases (RLKs) and/or receptor like proteins (RLPs) and transmit signals via the RLKs BAK1 and/or SOBIR1, as other well-known elicitors (Albert et al., 2015; Du et al., 2015; Postma et al., 2016; Franco-Orozco et al., 2017; Zhu et al., 2017; Nie et al., 2019; Nie et al., 2021). However, silencing of BAK1 or SOBIRl in tobacco did not block cell death development but instead, it resulted in induction of more severe and faster plant cell death (Fig. 9). Similar studies had also been reported that some apoplast elicitors could induce necrosis independent of BAK1 and/or SOBIR1, such as BcPG3 (Zhang et al., 2014b), TvEIX (Bar et al., 2010), VdEIX3 (Yin et al., 2021) and Fg12 (Yang et al., 2021). It is possible that BcPTP1 and its putative interacting RLP may coordinate with other unknown LRR-RLKs as co-receptors to transmit immune or death-inducing signals.

Subcellular localization analysis of BcPTP1 showed that the fluorescence signal of BcPTP1-GFP fusion protein is mainly observed in plant cell cytoplasmic vesicles and periphery of plasma membrane, but not the expected apoplastic localization (Fig. 3). This intracellular localization of BcPTP1 is unexpected given that an extracellular localization of the protein is necessary for the cytotoxic activity. Several studies showed that the internalization of certain secreted fungal proteins was dependent on the plasma membrane RLKs BAK1 and/or SOBIR1 (Robatzek, 2006a, Robatzek et al., 2006b; Chinchilla et al., 2007a, Chinchilla et al., 2007b; Liebrand et al., 2013; Wang et al., 2021b). Based on these studies and our findings, we proposed hypothetical model (Fig. 10) in which following secretion by the fungus, the BcPTP1 interacts in the apoplast with a plant RLK and/or RLP, and this complex transmits the phytotoxicity and defense signals. In parallel, the BcPTP1 protein might be also recognized by other plasma membrane receptor that cooperate with the BAK1 and SOBIR1 complex to mediate the internalization through cytoplasmic vesicles (Fig.

374 10). According to this model, silencing of the BAKl or SOBIRl genes blocks

375 BcPTP1internalization leading to accumulation of large amounts of BcPTP1 in apoplastic space and increased phytotoxicity.

\section{Acknowledgements}

379 This research was supported by the National Natural Science Foundation of 
381 Protection and Soil Science, Hubei Academy of Agricultural Sciences/Key 382 Laboratory of Integrated Pest Management on Crops in Central China, Ministry of 383 Agriculture, P.R. China/Hubei Key Laboratory of Crop Diseases, Insect Pests and 384 Weeds Control (Grant No 2018ZTSJJ14), and Hubei Province Agricultural Science and Technology Innovation Center Project (Grant No 2018-620-003-001).

386

387

388

389

390

391

392

393

394

395

396

397

398

399

400

401

402

403

404

405

406

407

408

409

410

411

412

413

414

415

416

417

418

419

\section{Author contributions}

WZ and WW: conceptualization of experiments and research plans; WZ, MY, QG, LG and WW: performing the experiments; RX, KB, CX and ZL: data and statistical analysis; WZ, AS, DJ and MW: funding acquisition; AS and WC: manuscript revision; WZ and WW: writing.

\section{Data availability}

Data of this study are all available within the paper and within its supplementary data published online. Further information may be obtained from the corresponding author.

\section{Materials and methods}

\section{Fungi, bacteria, plants and culture conditions}

The Botrytis cinerea wild type strain B05.10 and derived transformants used in this study were grown and maintained on PDA medium (Acumedia, MI, USA) at 22 under continuous fluorescent light supplemented with near-UV (black) light. Conidia of all B. cinerea strains were obtained from 7-days-old cultures. Escherichia coli strains Rosetta-gami (DE3) and JM109 (Shanghai Weidi Biotechnology, Shanghai, China) were respectively used for proteins expression and plasmid construction. Agrobacterium tumefaciens strain GV3101(pSoup) (Shanghai Weidi Biotechnology, Shanghai, China) was used for Agrobacterium-mediated transient expression of target proteins in plant leaves.

Tobacco (Nicotiana benthamiana), bean (Phaseolus vulgaris, genotype N9059), maize (Zea mays cv. Silver Queen) and tomato (Solanum lycopersicum cv. Hawaii 7981) plants were grown in a greenhouse under $16-\mathrm{h} / 8$-h intervals of $25^{\circ} \mathrm{C} / 22^{\circ} \mathrm{C}$, light/dark. Arabidopsis thaliana Columbia-0 was grown in a chamber under $16-\mathrm{h} / 8-\mathrm{h}$ intervals of $22^{\circ} \mathrm{C} / 20^{\circ} \mathrm{C}$, light/dark.

\section{Plasmid construction}

The bcptpl gene deletion construct Del-bcptpl was generated as described previously (Zhu et al., 2017) that the 5' (510 bp) and 3' (516 bp) flanks fragments of bcptpl gene were amplified and respectively cloned into the upstream and downstream regions of $h p h$ cassette using Gibson Assembly Master Mix kit (New 
420

421

422

423

424

425

426

427

428

429

430

431

432

433

434

435

436

437

438

439

440

441

442

443

444

445

446

447

448

449

450

451

452

453

454

455

456

457

458

England Biolabs, Massachusetts, USA). The overexpression plasmid OEBcPTP1$\mathrm{pH} 2 \mathrm{G}$ was generated that the full-length open reading frame of bcptpl was cloned into the $\mathrm{pH} 2 \mathrm{G}$ vector under the regulation of $B$. cinerea histone $\mathrm{H} 2 \mathrm{~B}$ promoter (NCBI identifier CP009806.1) and the endo- $\beta$-1,4-glucanase precursor terminator (NCBI identifier CP009807.1), as described previously (Zhu et al., 2017).

To transiently express the target protein in plant using agroinfiltration, the indicated sequences were cloned into binary plasmid pCNG between the $2 \times \mathrm{CaMV}$ $35 \mathrm{~S}$ promoter and NOS terminator (Yang et al., 2018), then transformed into A. tumefaciens strain GV3101. The E. coli protein expression vectors were constructed that the GFP and BcPTPl mature sequence without the signal peptide were, respectively, cloned into vector pET-N-GST-PreScission (Beyotime Biotechnology, Shanghai, China), then transformed into E. coli strain Rosetta-gami (DE3). All the primers used for plasmid construction were listed in Table S1.

\section{Manipulation of nucleic acids}

Total RNA of fungi and plant samples were isolated using RN03-RNApure Kit (Aidlab, Beijing, China), residual DNA was removed using DNase I (Thermo Scientific, MA, USA) according to manufacturers' protocols and stored at $-80^{\circ} \mathrm{C}$. The first strand cDNA of indicated sample was generated using RevertAid First Strand cDNA Synthesis Kit (Thermo Scientific, MA, USA). The reverse transcriptionquantitative PCR (RT-qPCR) was performed to analyze the gene expression profile using SYBR ${ }^{\circledR}$ Green Supermixes (Bio-Rad, CA, USA) and CFX96 Touch Real-Time PCR Detection System (Bio-Rad, CA, USA) according to manufacturer's instructions. The B. cinerea Bcgpdh gene and the $N$. benthamiana $N b E F 1 \alpha$ gene were, respectively, used as endogenous control genes for normalizing the expression levels of target genes as described previously (Zhu et al., 2017). Primers were designed across or flanking an intron (Supplementary Table S1). For each analyzed gene, RT-qPCR assays were repeated at least twice, each repetition with three independent replicates. The genomic DNA of indicated B. cinerea strains were isolated using Fungal Genomic DNA Purification Kit (Simgen, Hangzhou, China) according to the manufacturer's protocol.

\section{Bioinformatics analysis}

The NCBI (http://www.ncbi.nlm.nih.gov/) database was used to obtain homologous sequences of BcPTP1 from other pathogens using BLASTp analysis. The JGI (http://genome.jgi.doe.gov/Botci1/Botci1.home.html) database of B. cinerea was used to characterize $B$. cinerea genomic and transcriptomic sequences. The HMMSCAN (https://www.ebi.ac.uk/Tools/hmmer/search/hmmscan) was used to analyze the protein domain. The SignalP-5.0 Server 
459

460

461

462

463

464

465

466

467

468

469

470

471

472

473

474

475

476

477

478

479

480

481

482

483

484

485

486

487

488

489

490

491

492

493

494

495

496

497

(http://www.cbs.dtu.dk/services/SignalP/) was used to predict signal peptide sequence. The Clustal $\mathrm{X}$ and MEGA-X programs were used for protein alignments and phylogenetic tree generation with an unrooted neighbor-joining method. The I-TASSER (http://zhanglab.ccmb.med.umich.edu/I-TASSER/) was used to predict 3D structural model.

\section{Transformation, pathogenicity and cell wall stress tolerance assay of $B$. cinerea}

Genetic transformation of $B$. cinerea was performed as described previously (Ma et al., 2017). The bcptpl gene deletion mutants $\triangle B c P T P 1-1$ and $\triangle B c P T P 1-2$, overexpression strains OEBcPTP1-1 and OEBcPTP2, were confirmed using PCR and RT-qPCR.

Pathogenicity assays on the primary leaves of 9-days-old bean were performed as previously described (Zhu et al., 2017). Conidia of indicated B. cinerea strains were suspended in inoculation medium (Gamborg's B5 medium containing 2\% (w/v) glucose and $10 \mathrm{mM} \mathrm{KH} \mathrm{PO}_{4} / \mathrm{K}_{2} \mathrm{HPO}_{4}, \mathrm{pH} 6.4$ ), the conidia were diluted to $2 \times 10^{5}$ conidia/ml and leaves were inoculated with $7.5 \mu \mathrm{l}$ of spore suspension. Plants were incubated in a humid chamber at $22^{\circ} \mathrm{C}$ for $72 \mathrm{~h}$, and the lesion diameter was measured.

To determine the possible effect of BcPTP1 on stress tolerance and cell wall integrity, the indicated strains were inoculated on PDA plates supplemented with 0.5 $\mathrm{mg} / \mathrm{ml}$ Congo Red, $0.3 \mathrm{mg} / \mathrm{ml}$ Calcofluor White, $1 \mathrm{M}$ sorbitol, $1 \mathrm{M} \mathrm{NaCl}, 20 \mathrm{mM}$ $\mathrm{H}_{2} \mathrm{O}_{2}$ and $0.02 \%$ SDS at $22^{\circ} \mathrm{C}$, as described previously (Zhu et al., 2017).

\section{A. tumefaciens-mediated transient expression and Western blotting assay}

A. tumefaciens-mediated transient expression in $N$. benthamiana leaves was performed using agroinfiltration method as previously described (Zhu et al., 2017). For extraction of fungal or plant proteins, $0.2 \mathrm{~g}$ of tissue was ground to powder in liquid nitrogen, suspended in $1 \mathrm{ml}$ of cold lysis buffer (Beyotime Biotechnology, Shanghai, China), the samples were incubated on ice for $5 \mathrm{~min}$ and then centrifuged at $12,000 \mathrm{~g}$ for $10 \mathrm{~min}$ at $4^{\circ} \mathrm{C}$, the supernatant containing soluble proteins was collected. The supernatant proteins were then mixed with $5 \times$ SDS-PAGE sample buffer (Beyotime Biotechnology, Shanghai, China), denatured by boiling for $10 \mathrm{~min}$ at $100^{\circ} \mathrm{C}$ and then separated by SDS-PAGE electrophoresis and transferred onto PVDF membranes $(0.45 \mu \mathrm{m})$. Western blotting was analyzed using anti-GFP antibody (Beyotime Biotechnology, Shanghai, China).

\section{Expression and purification of recombinant BcPTP1 protein}

Expression of BcPTP1 and GFP recombinant proteins were performed in E. coli strain Rosetta-gami (DE3) as described previously (Zhu et al., 2017). Purification of 
498

499

500

501

502

503

504

505

506

507

508

509

510

511

512

513

514

515

516

517

518

519

520

521

522

523

524

525

526

527

528

529

530

531

532

533

534

535

536

recombinant proteins was performed using Glutathione Beads 4FF (Smart-Lifesciences, Changzhou, China) according to manufacturer's instructions. The proteins were cleaned using Amicon Ultra-4 Centrifugal Filter Devices (15 ml, 10 $\mathrm{kD}$; Merck Millipore) to remove the elution buffer, dissolved in phosphate-buffered saline (PBS) and stored at $-80^{\circ} \mathrm{C}$.

\section{Protein infiltration assay and induction of plant resistance by BcPTP1}

To test the phytotoxic activity of BcPTP1, leaves were infiltrated with recombinant protein solution. Plants were then kept in chamber at $25^{\circ} \mathrm{C}$ and photographed at 10- and 15-days after treatment.

To test the of BcPTP1 on plant defense response and sensitivity to infection, $N$. benthamiana leaves were infiltrated with $10 \mu \mathrm{g} / \mathrm{ml}$ protein solution. The infiltrated plants were kept in a greenhouse for $48 \mathrm{~h}$, and then the treated leaves were inoculated with $B$. cinerea and incubated for an additional $48 \mathrm{~h}$, or used for expression analysis of defense-related genes using RT-qPCR.

\section{VIGS in N. benthamiana}

To test whether BcPTP1-induced plant death is associated with $N b B A K 1$ or NbSOBIR1 in tobacco, VIGS was used to silence the expression of NbBAKl or NbSOBIR1 as described previously (Zhu et al., 2017). The plasmid pTRV2 :: GFP was used as the control. The expression level of $N b B A K 1$ or NbSOBIRl in gene silenced $N$. benthamiana was determined by RT-qPCR analysis. Then, the BcPTPI was expressed using A. tumefaciens-mediated transient expression in the leaves of NbBAK1 or NbSOBIR1 silenced $N$. benthamiana. The infiltrated plants were then kept in greenhouse at $25^{\circ} \mathrm{C}$ and the plant death development was photographed at 10 days and 15 days after infiltration.

\section{Subcellular localization analysis}

To analyze the subcellular localization of BcPTP1 in plant cells, the BcPTP1-GFP, BcPTP1 ${ }^{\triangle \mathrm{SP}}$-GFP, GFP and $\mathrm{SP}^{\mathrm{BcPTP} 1}$-GFP sequences were cloned into binary vector $\mathrm{pCNG}$ between the $2 \times \mathrm{CaMV} 35 \mathrm{~S}$ promoter and NOS terminator, respectively. Transient expression was carried out using agroinfiltration. $N$. benthamiana leaves epidermal cells were harvested 3 days after agroinfiltration and plasmolyzed in $0.75 \mathrm{M}$ sucrose solution, then the samples were imaged under confocal laser scanning microscope (Leica TCS SP8). Excitation wavelength of 488 $\mathrm{nm}$, emission wavelength of 495-510 nm was used to detect GFP, and 650-750 nm was used for chloroplast autofluorescence.

\section{Statistical analysis}


OriginPro 2021b (OriginLab Corporation, Northampton, MA, USA) was used for statistical tests. ANOVA (one-way, $\mathrm{P} \leq 0.01$ ) was used to analyze data significance. In all graphs, results were obtained from three to five independent experiments. Asterisks or different letters in the graphs indicate statistical differences at $\mathrm{P} \leq 0.01$.

\section{References}

1. Albert I, Böhm H, Albert M, Feiler CE, Imkampe J, Wallmeroth N, Brancato C, Raaymakers TM, Oome S, Zhang H, Krol E, Grefen C, Gust AA, Chai J, Hedrich R, Van den Ackerveken G, Nürnberger T. 2015. An RLP23-SOBIR1-BAK1 complex mediates NLP-triggered immunity. Nature Plants 1, 15140.

2. Albert I, Hua C, Nürnberger T, Pruitt RN, Zhang L. 2020. Surface sensor systems in plant immunity. Plant Physiology 182, 1582-1596.

3. Bar M, Sharfman M, Ron M, Avni A. 2010. BAK1 is required for the attenuation of ethylene-inducing xylanase (Eix)-induced defense responses by the decoy receptor LeEix1. Plant Journal 63, 791-800.

4. Bi K, Scalschi L, Jaiswal N, Mengiste T, Fried R, Sanz AB, Arroyo J, Zhu W, Masrati G, Sharon A. 2021. The Botrytis cinerea Crh1 transglycosylase is a cytoplasmic effector triggering plant cell death and defense response. Nature Communications 12, 2166.

5. Brunner F, Rosahl S, Lee J, Rudd JJ, Geiler C, Kauppinen S, Rasmussen G, Scheel D, Nürnberger T. 2002. Pep-13, a plant defense-inducing pathogen associated pattern from Phytophthora transglutaminases. EMBO Journal 21, 6681-6688.

6. Chinchilla D, Boller T, Robatzek S. 2007a. Flagellin signaling in plant immunity. Advances in Experimental Medicine and Biology 598, 358-371.

7. Chinchilla D, Zipfel C, Robatzek S, Kemmerling B, Nürnberger T, Jones JD, Felix G, Boller T. 2007b. A flagellin-induced complex of the receptor FLS2 and BAK1 initiates plant defence. Nature 448, 497-500.

8. Cui H, Tsuda K, Parker JE. 2015. Effector-triggered immunity: from pathogen perception to robust defense. Annual Review of Plant Biology 66, 487-511.

9. de Jonge R, van Esse HP, Maruthachalam K, Bolton MD, Santhanam P, Saber MK, Zhang Z, Usami T, Lievens B, Subbarao KV, Thomma BP. 2012. Tomato immune receptor Ve1 recognizes effector of multiple fungal pathogens uncovered by genome and RNA sequencing. Proceedings of the National Academy of Sciences, USA 109, 5110-5115.

10. Dean R, Van Kan JA, Pretorius ZA, Hammond-Kosack KE, Di Pietro A, Spanu PD, Rudd JJ, Dickman M, Kahmann R, Ellis J, Foster GD. 2012. The 
Top 10 fungal pathogens in molecular plant pathology. Molecular Plant Pathology 13, 414-430.

11. Denton-Giles M, McCarthy H, Sehrish T, Dijkwel Y, Mesarich CH, Bradshaw RE, Cox MP, Dijkwel PP. 2020. Conservation and expansion of a necrosis-inducing small secreted protein family from host-variable phytopathogens of the Sclerotiniaceae. Molecular Plant Pathology 21, 512-526.

12. Du J, Verzaux E, Chaparro-Garcia A, Bijsterbosch G, Keizer LC, Zhou J, Liebrand TW, Xie C, Govers F, Robatzek S, van der Vossen EA, Jacobsen E, Visser RG, Kamoun S, Vleeshouwers VG. 2015. Elicitin recognition confers enhanced resistance to Phytophthora infestans in potato. Nature Plants 1, 15034.

13. Eizner E, Ronen M, Gur Y, Gavish A, Zhu W, Sharon A. 2017. Characterization of Botrytis-plant interactions using PathTrack@: an automated system for dynamic analysis of disease development. Molecular Plant Pathology 18, 503-512.

14. Fliegmann J, Mith $\square$ fer A, Wanner G, Ebel J. 2004. An ancient enzyme domain hidden in the putative $\beta$-glucan elicitor receptor of soybean may play an active part in the perception of pathogen-associated molecular patterns during broad host resistance. Journal of Biological Chemistry 279, 1132-1140.

15. Franco-Orozco B, Berepiki A, Ruiz O, Gamble L, Griffe LL, Wang S, Birch PRJ, Kanyuka K, Avrova A. 2017. A new proteinaceous pathogen-associated molecular pattern (PAMP) identified in Ascomycete fungi induces cell death in Solanaceae. New Phytologist 214, 1657-1672.

16. Frías M, González C, Brito N. 2011. BcSpl1, a cerato-platanin family protein, contributes to Botrytis cinerea virulence and elicits the hypersensitive response in the host. New Phytologist 192, 483-495.

17. Frías M, González M, González C, Brito N. 2016. BcIEB1, a Botrytis cinerea secreted protein, elicits a defense response in plants. Plant Science 250, 115-124.

18. Gao Y, Faris JD, Liu Z, Kim YM, Syme RA, Oliver RP, Xu SS, Friesen TL. 2015. Identification and characterization of the SnTox6-Snn6 interaction in the Parastagonospora nodorum-wheat pathosystem. Molecular Plant-Microbe Interactions 28, 615-625.

19. Gui YJ, Chen JY, Zhang DD, Li NY, Li TG, Zhang WQ, Wang XY, Short DPG, Li L, Guo W, Kong ZQ, Bao YM, Subbarao KV, Dai XF. 2017. Verticillium dahliae manipulates plant immunity by glycoside hydrolase 12 proteins in conjunction with carbohydrate-binding module 1. Environmental Microbiology 19, 1914-1932.

20. Heard S, Brown NA, Hammond-Kosack K. 2015. An interspecies comparative analysis of the predicted secretomes of the necrotrophic plant pathogens Sclerotinia sclerotiorum and Botrytis cinerea. PLoS ONE 10, e0130534. 
614 21. Jones JDG, Dangl JL. 2006. The plant immune system. Nature 444, 323-329.

615

616

617

618

619

620

621

622

623

624

625

626

627

628

629

630

631

632

633

634

635

636

637

638

639

640

641

642

643

644

645

646

647

648

649

650

651

652

22. Kanneganti T, Huitema E, Cakir C, Kamoun S. 2006. Synergistic interactions of the plant cell death pathways induced by Phytophthora infestans Nep1-like protein PiNPP1.1 and INF1 elicitin. Molecular Plant-Microbe Interactions 19, 854-863.

23. Kim K-T, Jeon J, Choi J, Cheong K, Song H, Choi G, Kang S, Lee Y-H. 2016. Kingdom-wide analysis of fungal small secreted proteins (SSPs) reveals their potential role in host association. Frontiers in Plant Science 7, 186.

24. Li Y, Han Y, Qu M, Chen J, Chen X, Geng X, Wang Z, Chen S. 2020. Apoplastic cell death-inducing proteins of filamentous plant pathogens: roles in plant-pathogen interactions. Frontiers in Genetics 11, 661.

25. Liebrand TW, van den Berg GC, Zhang Z, Smit P, Cordewener JH, America AH, Sklenar J, Jones AM, Tameling WI, Robatzek S, Thomma BP, Joosten MH. 2013. Receptor-like kinase SOBIR1/EVR interacts with receptor-like proteins in plant immunity against fungal infection. Proceedings of the National Academy of Sciences, USA 110, 10010-10015.

26. Lo Presti L, Lanver D, Schweizer G, Tanaka S, Liang L, Tollot M, Zuccaro A, Reissmann S, Kahmann R. 2015. Fungal effectors and plant susceptibility. Annual Review of Plant Biology 66, 513-545.

27. Lorang J, Kidarsa T, Bradford CS, Gilbert B, Curtis M, Tzeng SC, Maier CS, Wolpert TJ. 2012. Tricking the guard: exploiting plant defense for disease susceptibility. Science 338, 659-662.

28. Lu S, Edwards MC. 2016. Genome-wide analysis of small secreted cysteine-rich proteins identifies candidate effector proteins potentially involved in Fusarium graminearum-wheat interactions. Phytopathology 106, 166-176.

29. Ma L, Salas O, Bowler K, Oren-Young L, Bar-Peled M, Sharon A. 2017. Genetic alteration of UDP-rhamnose metabolism in Botrytis cinerea leads to the accumulation of UDP-KDG that adversely affects development and pathogenicity. Molecular Plant Pathology 18, 263-275.

30. Ma Z, Song T, Zhu L, Ye W, Wang Y, Shao Y, Dong S, Zhang Z, Dou D, Zheng X, Tyler BM, Wang Y. 2015. A Phytophthora sojae glycoside hydrolase 12 protein is a major virulence factor during soybean infection and is recognized as a PAMP. Plant Cell 27, 2057-2072.

31. Ma Z, Zhu L, Song T, Wang Y, Zhang Q, Xia Y, Qiu M, Lin Y, Li H, Kong L, Fang Y, Ye W, Wang Y, Dong S, Zheng X, Tyler BM, Wang Y. 2017. A paralogous decoy protects Phytophthora sojae apoplastic effector PsXEG1 from a host inhibitor. Science 355, 710-714.

32. Marianayagam NJ, Sunde M, Matthews JM. 2004. The power of two: protein dimerization in biology. Trends in Biochemical Sciences 29, 618-625. 
653

654

655

656

657

658

659

660

661

662

663

664

665

666

667

668

669

670

671

672

673

674

675

676

677

678

679

680

681

682

683

684

685

686

687

688

689

690

691

33. Nie J, Yin Z, Li Z, Wu Y, Huang L. 2019. A small cysteine-rich protein from two kingdoms of microbes is recognized as a novel pathogen-associated molecular pattern. New Phytologist 222, 995-1011.

34. Nie J, Zhou W, Liu J, Tan N, Zhou JM, Huang L. 2021. A receptor-like protein from Nicotiana benthamiana mediates VmE02 PAMP-triggered immunity. New Phytologist 229, 2260-2272.

35. Oome S, Raaymakers TM, Cabral A, Samwel S, B $\square$ hm H, Albert I, Nürnberger T, Van den Ackerveken G. 2014. Nep1-like proteins from three kingdoms of life act as a microbe-associated molecular pattern in Arabidopsis. Proceedings of the National Academy of Sciences, USA 111, 16955-16960.

36. Postma J, Liebrand TWH, Bi G, Evrard A, Bye RR, Mbengue M, Kuhn H, Joosten MHAJ, Robatzek S. 2016. Avr4 promotes Cf-4 receptor-like protein association with the BAK1/SERK3 receptor-like kinase to initiate receptor endocytosis and plant immunity. New Phytologist 210, 627-642.

37. Rep M. 2005. Small proteins of plant-pathogenic fungi secreted during host colonization. FEMS Microbiology Letters 253, 19-27.

38. Robatzek S. 2006a. Vesicle trafficking in plant immune responses. Cell Microbiology 9, 1-8.

39. Robatzek S, Chinchilla D, Boller T. 2006b. Ligand-induced endocytosis of the pattern recognition receptor FLS2 in Arabidopsis. Genes and Development 20, 537-542.

40. Rotblat B, Enshell-Seijffers D, Gershoni JM, Schuster S, Avni A. 2002. Identification of an essential component of the elicitation active site of the EIX protein elicitor. Plant Journal 32, 1049-1055.

41. Sevier CS, Kaiser CA. 2002. Formation and transfer of disulphide bonds in living cells. Nature Reviews Molecular Cell Biology 3, 836-847.

42. Shao D, Smith DL, Kabbage M, Roth MG. 2021. Effectors of plant necrotrophic fungi. Frontiers in Plant Science 12, 687713.

43. Shinya T, Nakagawa T, Kaku H, Shibuya N. 2015. Chitin-mediated plant-fungal interactions: catching, hiding and handshaking. Current Opinion in Plant Biology 26, 64-71.

44. Stergiopoulos I, de Wit PJGM. 2009. Fungal effector proteins. Annual Review of Phytopathology 47, 233-263.

45. Thomma BP, Nürnberger T, Joosten MH. 2011. Of PAMPs and effectors: the blurred PTI-ETI dichotomy. Plant Cell 23, 4-15.

46. van Ooijen G, van den Burg HA, Cornelissen BJ, Takken FL. 2007. Structure and function of resistance proteins in solanaceous plants. Annual Review of Phytopathology 45, 43-72.

47. Veloso J, van Kan JAL. 2018. Many shades of grey in Botrytis-host plant 
692

693

694

695

696

697

698

699

700

701

702

703

704

705

706

707

708

709

710

711

712

713

714

715

716

717

718

719

720

721

722

723

724

725

726

727

728

729

730

interactions. Trends in Plant Science 23, 613-622.

48. Wang D, Chen J-Y, Song J, Li J-J, Klosterman SJ, Li R, Kong Z-Q, Subbarao KV, Dai X-F, Zhang D-D. 2021b. Cytotoxic function of xylanase VdXyn4 in the plant vascular wilt pathogen Verticillium dahliae. Plant Physiology https://doi.org/10.1093/plphys/kiab274

49. Wang Y, Xu Y, Sun Y, Wang H, Qi J, Wan B, Ye W, Lin Y, Shao Y, Dong S, Tyler BM, Wang Y. 2018. Leucine-rich repeat receptor-like gene screen reveals that Nicotiana RXEG1 regulates glycoside hydrolase 12 MAMP detection. Nature Communications 9, e594.

50. Wang S, Xing R, Wang Y, Shu H, Fu S, Huang J, Paulus JK, Schuster M, Saunders DGO, Win J, Vleeshouwers V, Wang Y, Zheng X, van der Hoorn RAL, Dong S. 2021a. Cleavage of a pathogen apoplastic protein by plant subtilases activates host immunity. New Phytologist 229, 3424-3439.

51. Yang B, Wang Y, Tian M, Dai K, Zheng W, Liu Z, Yang S, Liu X, Shi D, Zhang H, Wang Y, Ye W, Wang Y. 2021. Fg12 ribonuclease secretion contributes to Fusarium graminearum virulence and induces plant cell death. Journal of Integrative Plant Biology 63, 365-377.

52. Yang G, Tang L, Gong Y, Xie J, Fu Y, Jiang D, Li G, Collinge DB, Chen W, Cheng J. 2018. A cerato-platanin protein SsCP1 targets plant PR1 and contributes to virulence of Sclerotinia sclerotiorum. New Phytologist 217, 739-755.

53. Yin Z, Wang N, Pi L, Li L, Duan W, Wang X, Dou D. 2021. Nicotiana benthamiana LRR-RLP NbEIX2 mediates the perception of an EIX-like protein from Verticillium dahliae. Journal of Integrative Plant Biology 63, 949-960.

54. Zhang H, Wu Q, Cao S, Zhao T, Chen L, Zhuang P, Zhou X, Gao Z. 2014a. A novel protein elicitor (SsCut) from Sclerotinia sclerotiorum induces multiple defense responses in plants. Plant Molecular Biology 86, 495-511.

55. Zhang L, Kars I, Essenstam B, Liebrand TWH, Wagemakers L, Elberse J, Tagkalaki P, Tjoitang D, van den Ackerveken G, van Kan JAL. 2014b. Fungal endopolygalacturonases are recognized as microbe-associated molecular patterns by the Arabidopsis receptor-like protein RESPONSIVENESS TO BOTRYTIS POLYGALACTURONASES1. Plant Physiology 164, 352-364.

56. Zhang L, Ni H, Du X, Wang S, Ma XW, Nürnberger T, Guo HS, Hua C. 2017. The Verticillium-specific protein VdSCP7 localizes to the plant nucleus and modulates immunity to fungal infections. New Phytologist 215, 368-381.

57. Zhu W, Ronen M, Gur Y, Minz-Dub A, Masrati G, Ben-Tal N, Sharon I, Savidor A, Eizner E, Valerius O, Braus G, Bowler K, Bar-Peled M, Sharon A. 2017. BcXYG1, a secreted xyloglucanase from Botrytis cinerea, triggers both cell death and plant immune responses. Plant Physiology 175, 438-456. 
A

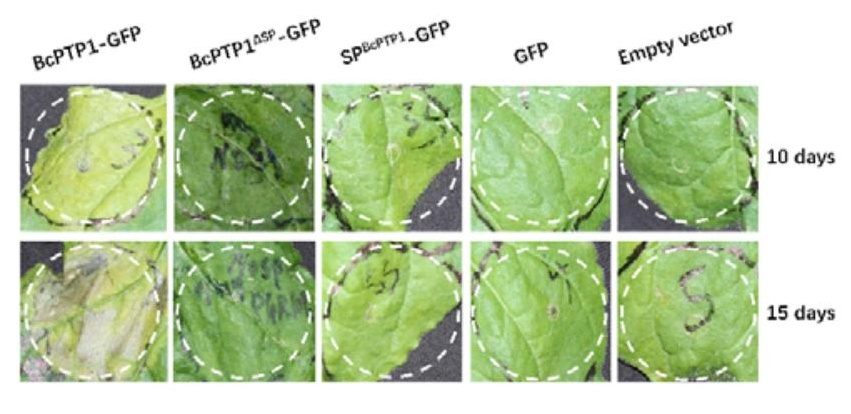

B

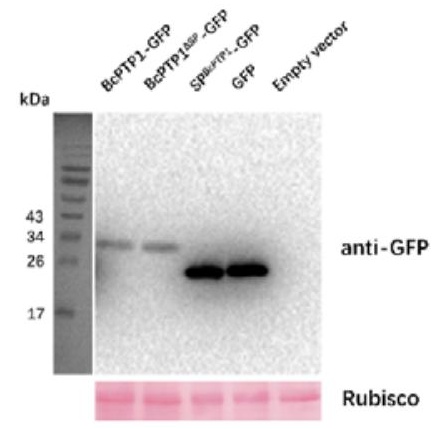

Fig. 1. BcPTP1 is a phytotoxic secreted protein. (A) Images of $N$. benthamiana leaves 10 days and 15 days after agroinfiltration with A. tumefaciens strains carrying the indicated constructs. The A. tumefaciens carrying the empty vector was used as control. BcPTP1-GFP, BcPTP1 fused with GFP at the C-terminus; BcPTP1 ${ }^{\triangle \mathrm{SP}}$-GFP, $\mathrm{BcPTP} 1$ without signal peptide fused with GFP at the C-terminus; $\mathrm{SP}^{\mathrm{BcPTP} 1}$-GFP, GFP fused with the signal peptide of BcPTP1. (B) Immunoblot analysis of proteins from $N$. benthamiana leaves transiently expressing the indicated constructs. Top panel, immunoblot using anti-GFP antibody; bottom panel, staining of the Rubisco large subunit with Ponceau S. 
A
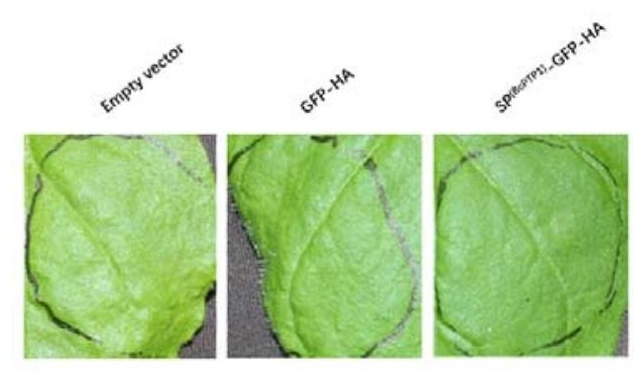

C

B

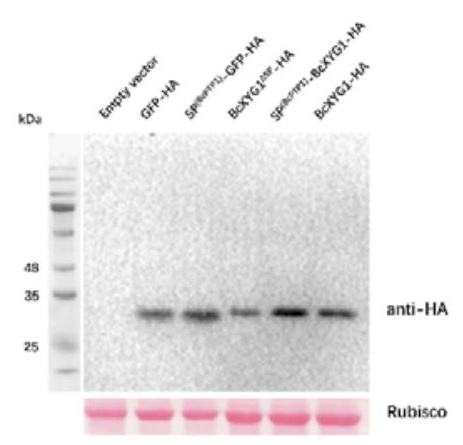

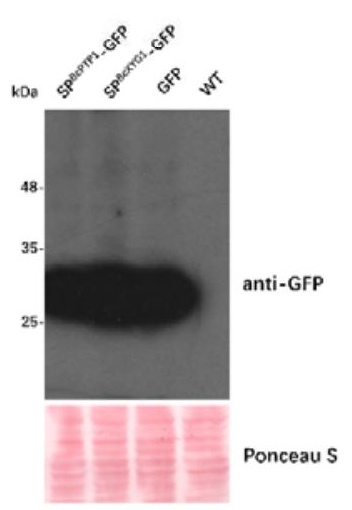

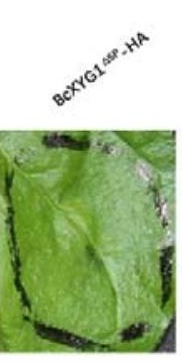

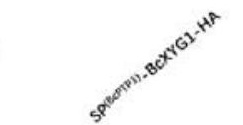
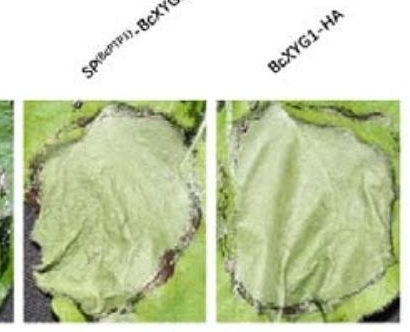

D

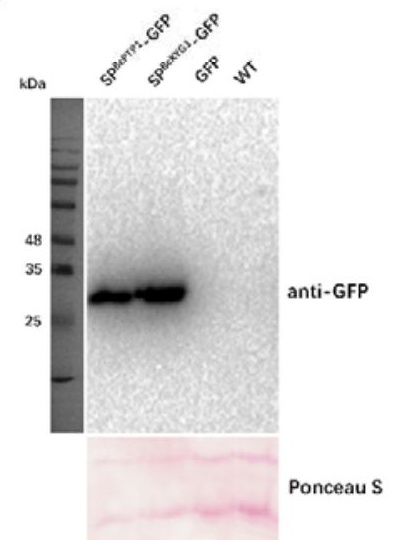

Fig. 2. BcPTP1 signal peptide possesses secretion function. (A) Images of $N$. benthamiana leaves 5 days after agroinfiltration with A. tumefaciens strains carrying the indicated constructs. The A. tumefaciens carrying the empty vector was used as control. GFP-HA, HA-tagged GFP; SP ${ }^{(\mathrm{BcPTP} 1)}$-GFP-HA, HA-tagged GFP with the signal peptide of BcPTP1; BcXYG1 ${ }^{\triangle S P}$-HA, HA-tagged BcXYG1 lacking the native signal peptide; $\mathrm{SP}^{(\mathrm{BcPTP1})}-\mathrm{BcXYG1-HA}$, HA-tagged $\mathrm{BcXYG1}$ with its native signal peptide replaced with the BcPTP1 signal peptide; BcXYG1-HA, HA-tagged BcXYG1 with its native signal peptide. (B) Immunoblot analysis of proteins from $N$. benthamiana leaves transiently expressing the indicated constructs. Top panel, immunoblot using anti-HA antibody; bottom panel, staining of the Rubisco large subunit with Ponceau S. (C-D) The indicated genes were expressed under the regulation of $B$. cinerea histone $\mathrm{H} 2 \mathrm{~B}$ promoter (NCBI identifier CP009806.1) and the endo- $\beta$-1,4-glucanase precursor terminator (NCBI identifier CP009807.1). The $B$. cinerea strains were cultured in PDB medium for $72 \mathrm{~h}$, then the culture filtrate was collected and purified by filtration. (C) Immunoblot analysis of total mycelia proteins from indicated $B$. cinerea strains. Top panel, immunoblot using anti-GFP antibody; bottom panel, Ponceau $S$ staining of the total mycelia proteins. (D) Immunoblot analysis of culture filtrate proteins from indicated $B$. cinerea strains. Top panel, immunoblot using anti-GFP antibody; bottom panel, Ponceau $\mathrm{S}$ staining of the secretory proteins. $\mathrm{SP}^{\mathrm{BcPTP}}$-GFP, GFP fused with the signal peptide of BcPTP1; $\mathrm{SP}^{\mathrm{BcXYG1}}$-GFP, GFP fused with the signal peptide of $\mathrm{BcXYG1;}$ GFP, GFP 
overexpression strain; WT, wild type strain.

GFP

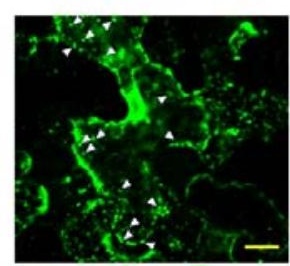

Chloroplast
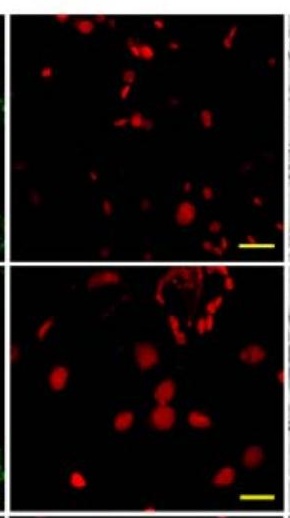

BCPTP1 $^{\triangle S P}$ _GFP

SPBPTP1-GFP
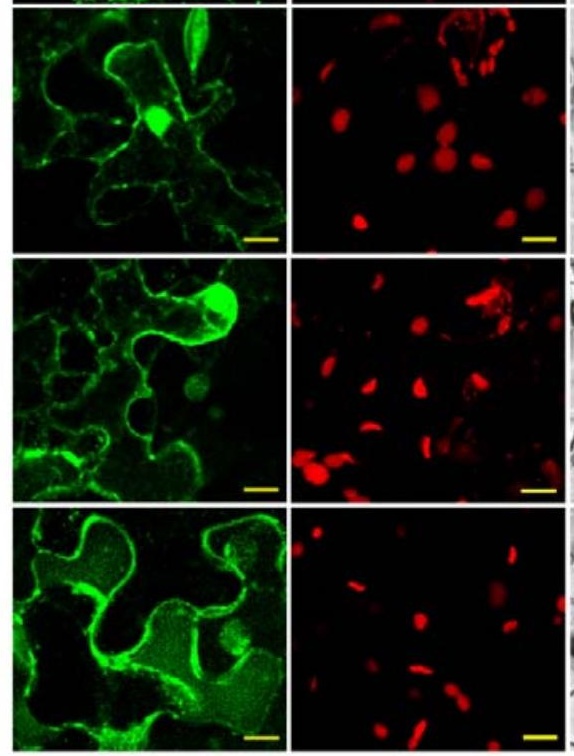

Bright field
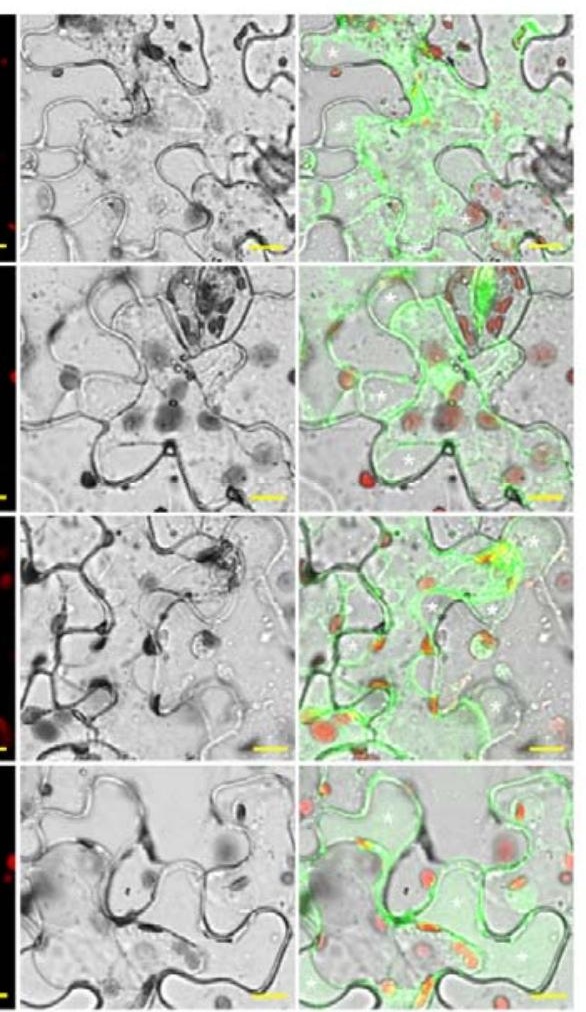

\section{Fig. 3. Subcellular localization of BcPTP1 in N. benthamiana epidermal cells.}

Leaves epidermal cells were harvested 3 days after agroinfiltration and plasmolyzed in $0.75 \mathrm{M}$ sucrose solution, then the samples were scanned by confocal laser scanning microscope. BcPTP1-GFP, GFP fused to full length BcPTP1; BcPTP1 ${ }^{\triangle \mathrm{SP}}$-GFP, GFP fused to BcPTP1 without signal peptide; $\mathrm{SP}^{\mathrm{BcPTP} 1}-\mathrm{GFP}$, GFP fused to the BcPTP1 signal peptide. The white arrows indicate the internalized cytoplasmic vesicle. White asterisks mark apoplastic space of plant cells. Bars $=10 \mu \mathrm{m}$. 
798

799

800

801

802

803

804

805

806

807

808

809

810

811

812

813

814

815

816

817

818

819

820

821

822

823

824

825
A

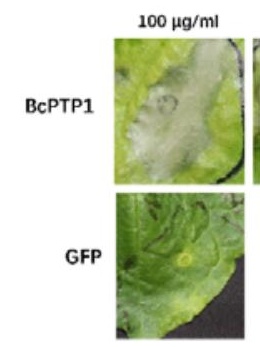

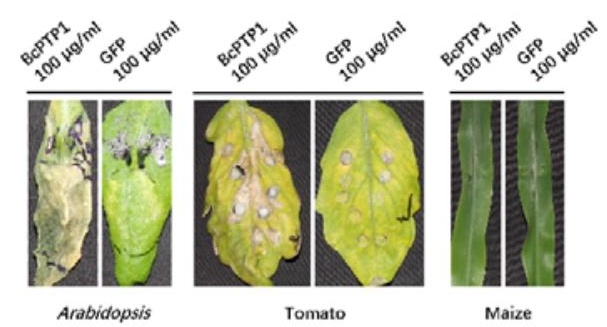

Arabidopsis

Fig. 4. The BcPTP1 protein is phytotoxic to dicot but not monocot plant species.

Proteins were produced in E. coli, purified and suspended in phosphate-buffered saline (PBS). (A) Response of $N$. benthamiana leaves infiltrated with different concentrations of BcPTP1 at 15 days after treatment. (B) Response of Arabidopsis, tomato and maize leaves infiltrated with $100 \mu \mathrm{g} / \mathrm{ml}$ protein solution.

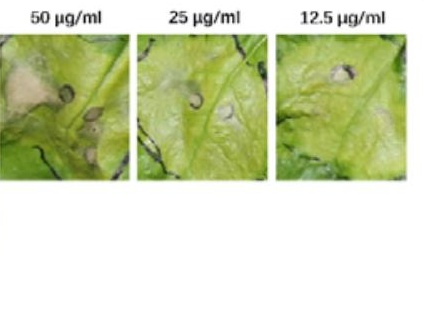


A
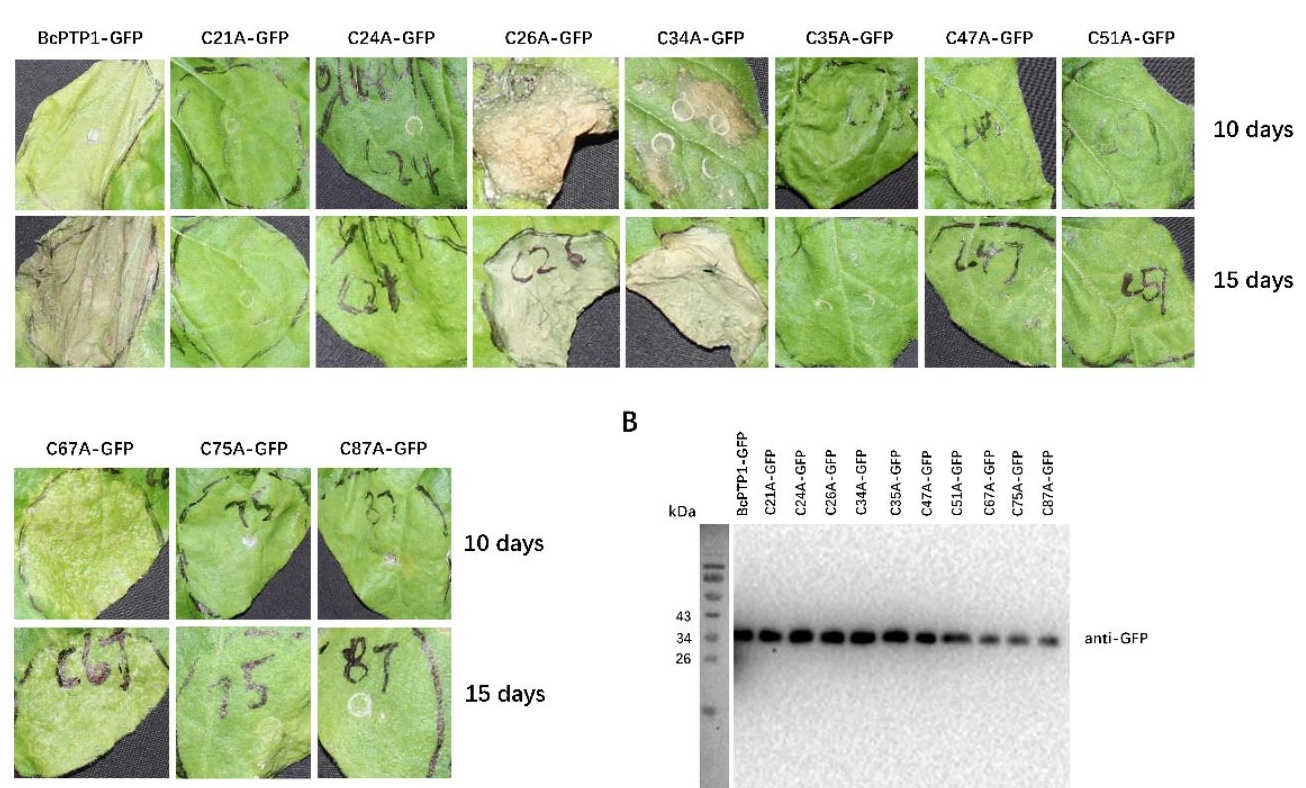

B

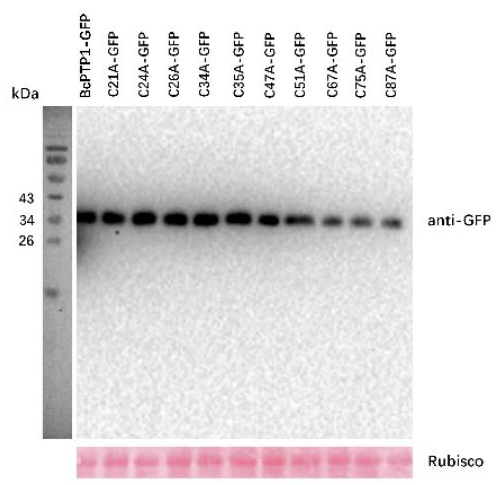

Fig. 5. Specific cysteine residues necessary for BcPTP1 phytotoxicity. (A) Images of $N$. benthamiana leaves 10 days and 15 days after transient agroinfiltration. (B) Immunoblot analysis of proteins from $N$. benthamiana leaves transiently expressing the cysteine residue mutant constructs. Top panel, immunoblot using anti-GFP antibody; Bottom panel, staining of the Rubisco large subunit with Ponceau S.

833

834

835

836

837

838

839

840

841

842 


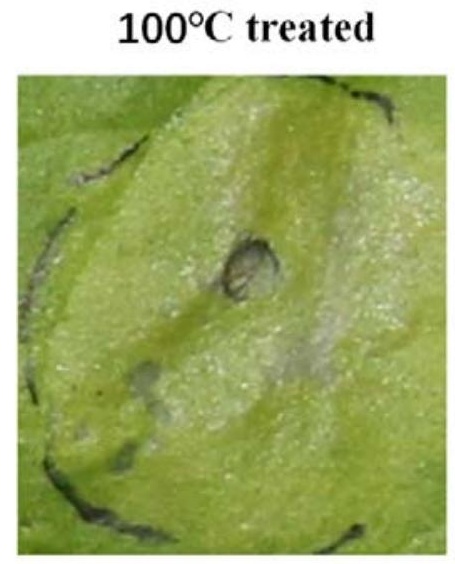

$25^{\circ} \mathrm{C}$ treated

844 Fig. 6. Phytotoxicity of BcPTP1 is partially heat-stable. Images of $N$. benthamiana

845 leaves 15 days after infiltration with $50 \mu \mathrm{g} / \mathrm{ml}$ of heat-treated $(100 \square$ treated for 30

$846 \mathrm{~min}$ ) and native (25 $\square$ treated for $30 \mathrm{~min}$ ) BcPTP1 protein.

847

848

849

850

851

852

853

854

855

856

857

858

859

860

861

862

863

864

865

866

867

868

869

870 


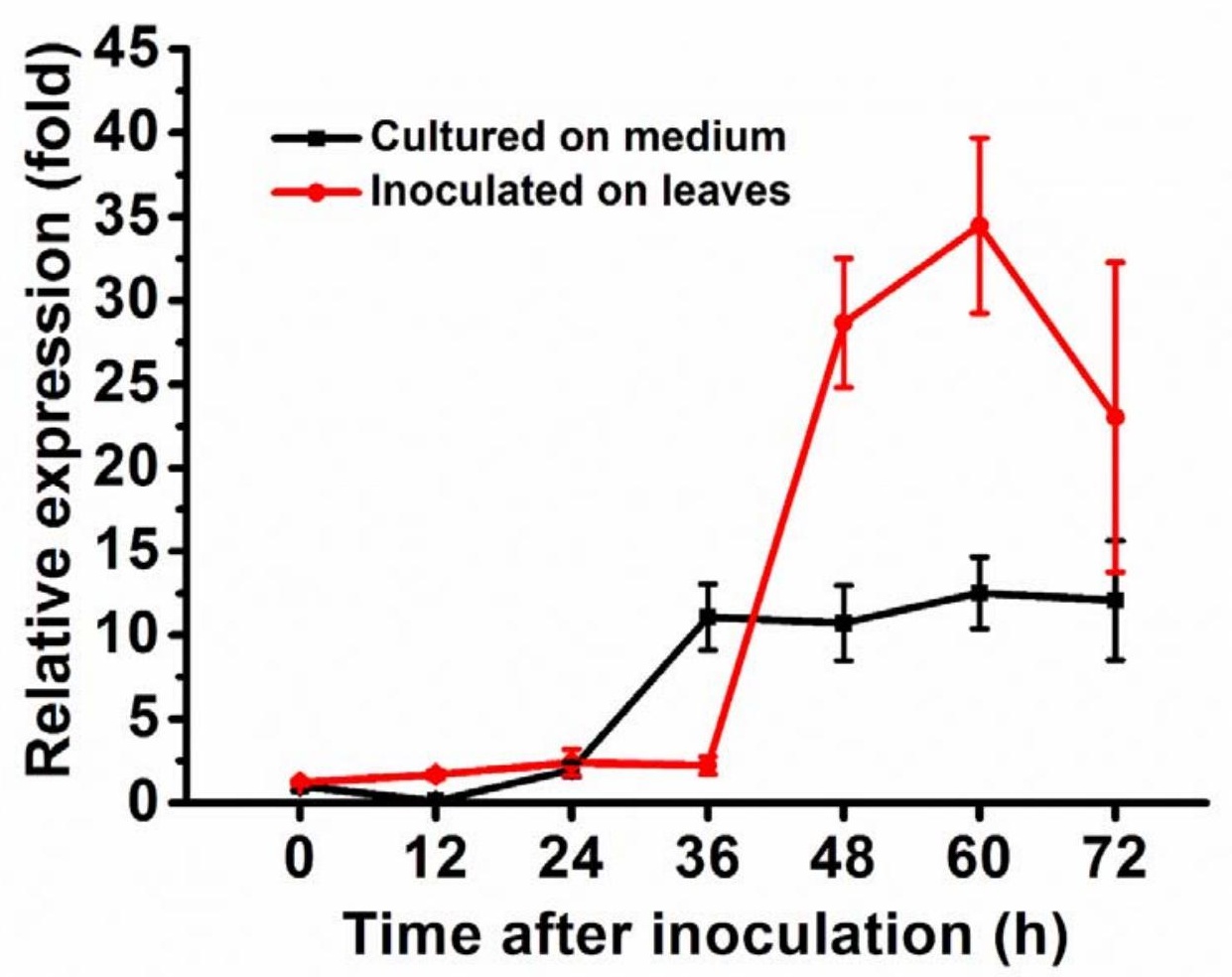

871

872 Fig. 7. Expression of the BcPTP1 gene is up-regulated at late infection stage.

873 Bean leaves (red line) or Gamborg's B5 medium (black line) were inoculated with $B$.

874 cinerea conidia, and expression levels of the BcPTPl gene were evaluated by

875 RT-qPCR. The expression level of BcPTP1 inoculated on plant or in Gamborg's B5

876 medium at $0 \mathrm{~h}$ was set as 1 , and relative transcript levels were calculated using the

877 comparative $\mathrm{Ct}$ method. Transcript levels of the B. cinerea Bcgpdh gene were used to

878 normalize different samples. Data represent means and standard deviations of three

879 independent replications.

880

881

882

883

884

885

886

887 
A
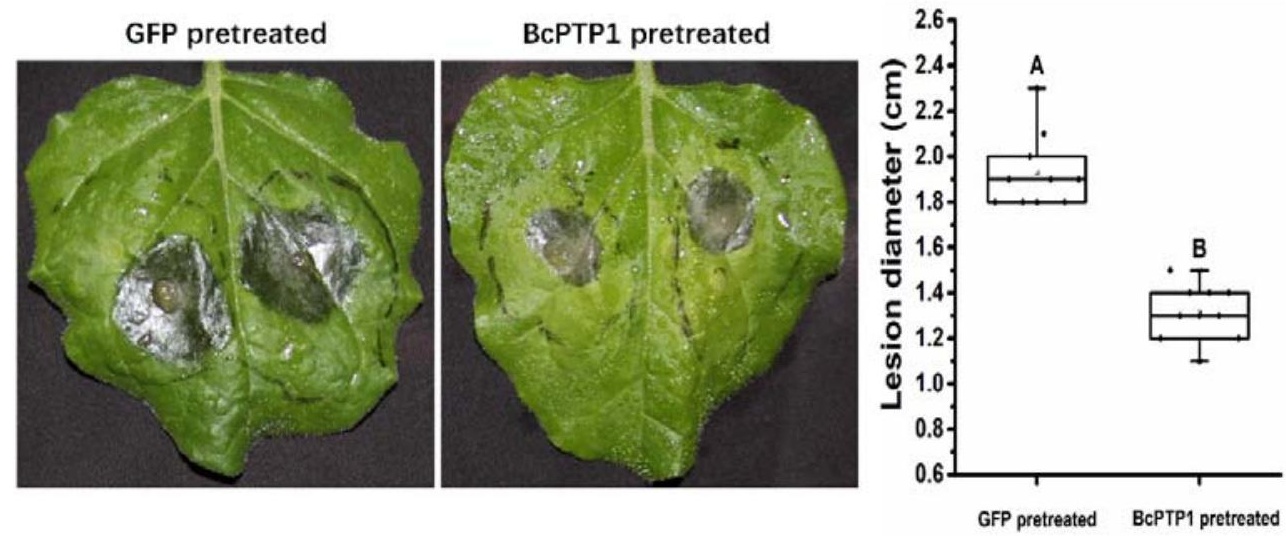

B
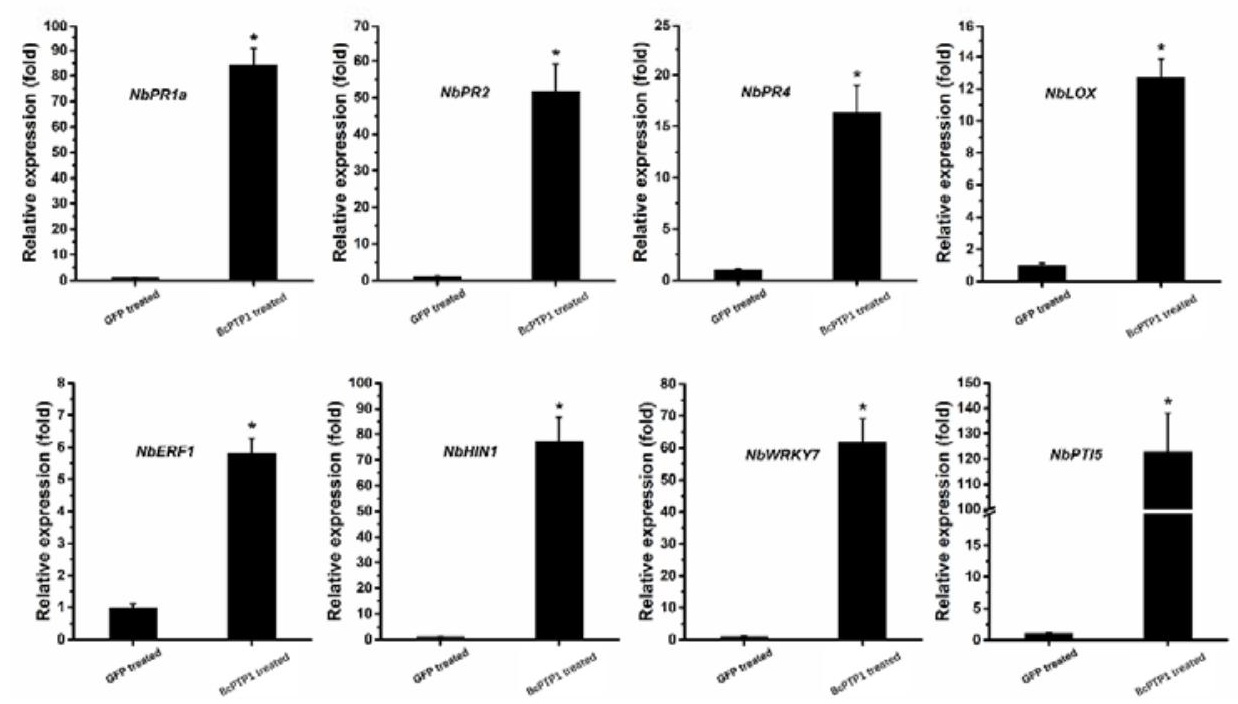

Fig. 8. BcPTP1 induces resistance in tobacco. (A) $N$. benthamiana leaves were infiltrated with $10 \mu \mathrm{g} / \mathrm{ml}$ purified BcPTP1 or GFP protein. After 2 days, the infiltrated leaves were inoculated with $B$. cinerea in a humid chamber. The lesions were photographed and measured at 48 hpi. Data were obtained from three independent experiments with total 10 samples. In box plots, whiskers indicate the minimum and maximum values, the line indicates the median, the box boundaries indicate the upper (25th percentile) and lower (75th percentile) quartiles, all data are plotted as black dots. Different letters in the graph indicate statistical differences at $\mathrm{P} \leq 0.01$ using ANOVA (one-way). (B) Relative expression levels of defense-related genes from tobacco leaves that treated with BcPTP1 or GFP for $48 \mathrm{~h}$ were determined by RT-qPCR analysis. The expression level of indicated genes in GFP-treated leaves were set as 1. The expression level of the tobacco NbEFla gene was used to normalize different samples. Data represent means and standard deviations of three 
0.01 using ANOVA (one-way).

A
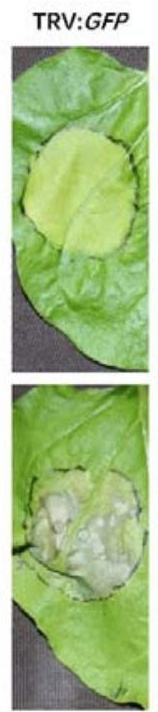
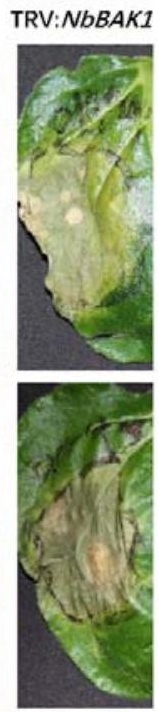
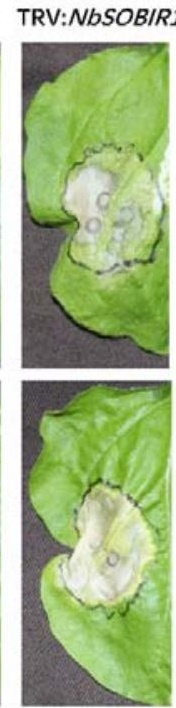

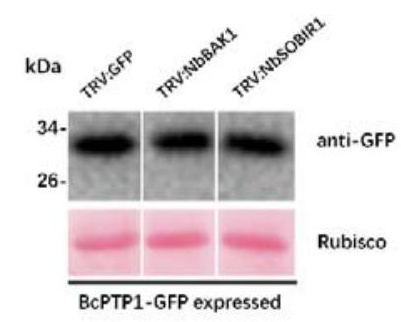

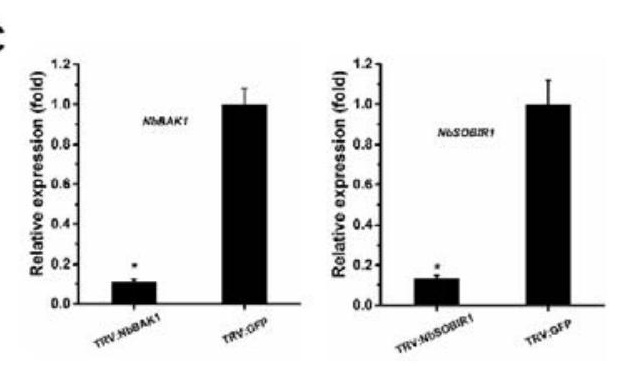

905

906

907

908

909

910

911

912

913

914

915

916

917

918

919

920

921

922
B

C

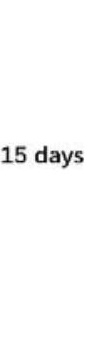

Fig. 9. BAK1 and SOBIR1 negatively regulate the death-inducing activity of BcPTP1 in $N$. benthamiana. TRV-based VIGS vectors were used to initiate silencing of NbBAK1 (TRV : NbBAKl) and NbSOBIRl (TRV : NbSOBIRl). TRV : GFP was used as a control virus treatment in these experiments. (A) Three weeks after initiation of VIGS, BcPTP1-GFP was transiently expressed in the gene-silenced leaves using agroinfiltration. Leaves were photographed 10 and 15 days after treatment. (B) Immunoblot analysis of proteins from indicated $N$. benthamiana leaves transiently expressing BcPTP1-GFP. Top panel: BcPTP1-GFP was detected using anti-GFP antibody; bottom panel, staining of the Rubisco large subunit with Ponceau S. (C) $N b B A K 1$ and NbSOBIR1 expression levels in gene-silenced tobacco leaves were determined by RT-qPCR analysis. Expression level in control plants (TRV : GFP) was set as 1. NbEF1 $\alpha$ was used as an endogenous control. Data represent means and standard deviations from three biological replicates. Asterisks indicate significant differences $(\mathrm{P} \leq 0.01)$. 


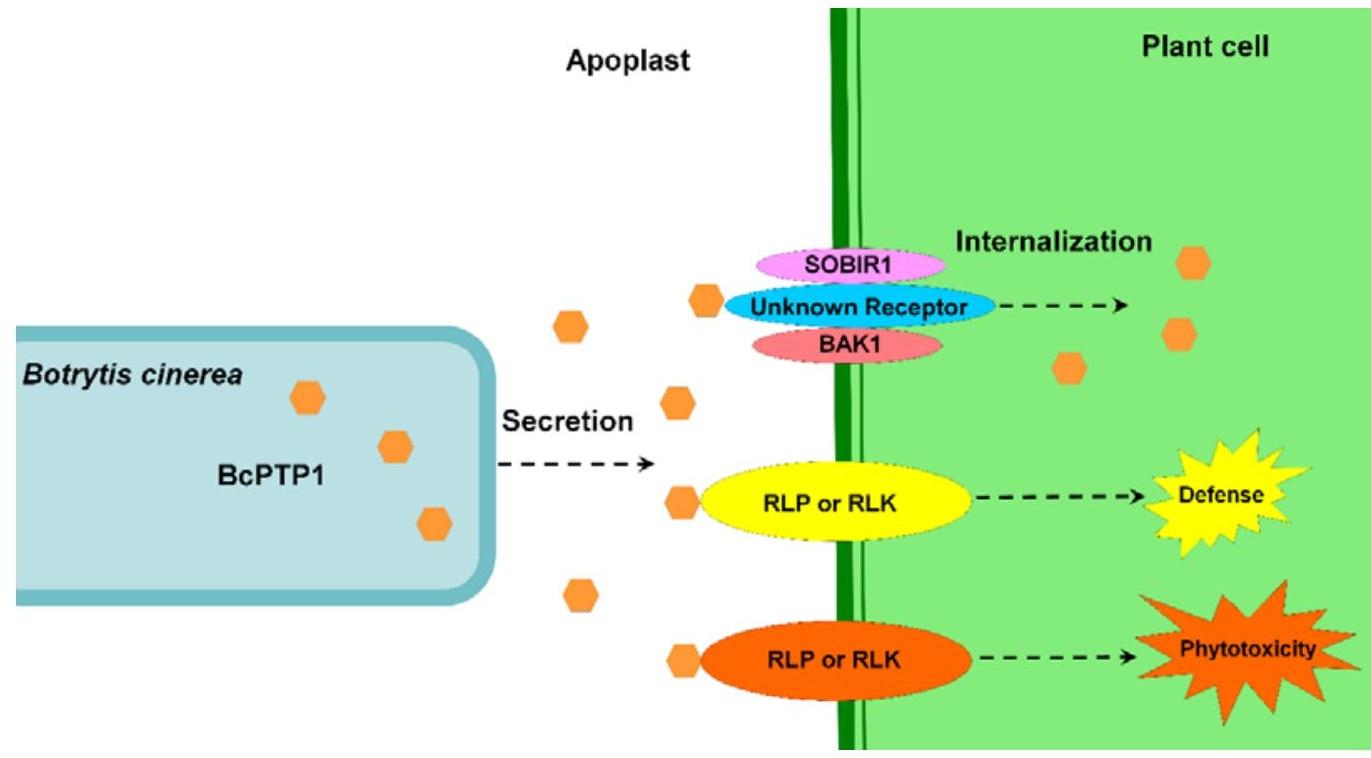

926

927

Fig. 10. The model illustrating the internalization of BcPTP1 mediated by BAK1

928 and SOBIR1, and the defense and phytotoxicity induced by BcPTP1 though

929 RLPs or PLKs in plant cell. BcPTP1 is secreted from B. cinerea hyphae into

930 apoplastic space to interact with plants RLKs and/or RLPs and transmit the phytotoxicity and defense signals. BcPTP1 can also be recognized by other unknown receptor that cooperate with BAK1 and SOBIR1 complex to mediate the

933 internalization.

934

935

936

937

938

939

940

941

942

943

944 
$72 \mathrm{KD}$

$55 \mathrm{KD}$

$43 \mathrm{KD}$

$34 \mathrm{KD}$

Fig. S1. Expression of recombinant proteins. SDS-PAGE analysis of purified GST- 
A

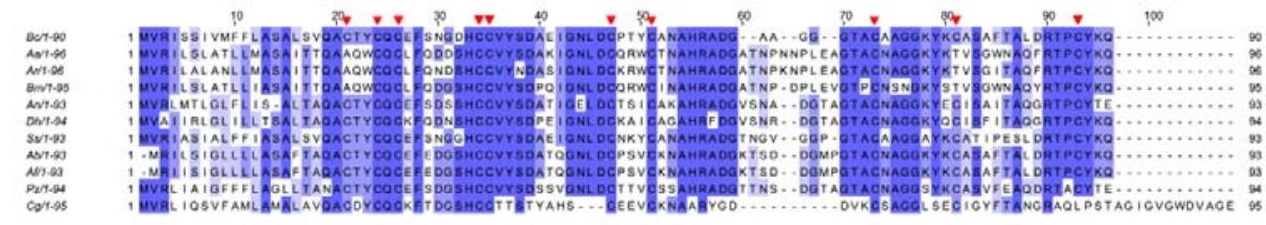

B

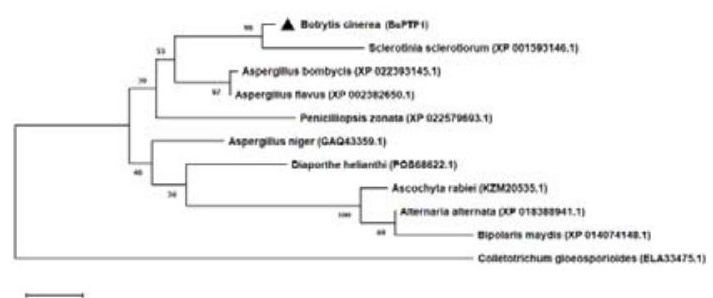

C

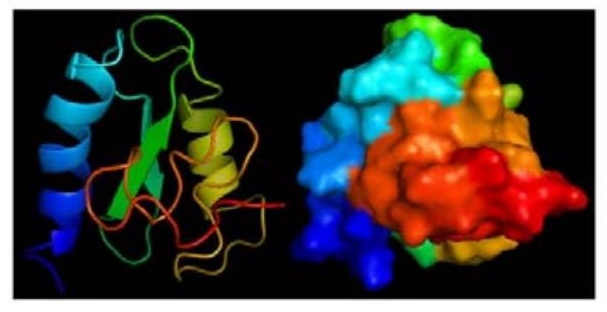

961

962

963

964

965

966

967

968

969

970

971

972

973

974

975

976

977

978

979

980

981

982

983

Fig. S2. Sequence similarities between BcPTP1 and its homologs. (A) Multiple sequence alignment of BcPTP1 and its homologs. Full-length protein sequences were aligned using Clustal $W$ and the alignment was edited using Jalview. The red triangles show the cysteine residues. Intensity of blue shading reflects the level of amino acid identity at each position. Bc: B. cinerea BcPTP1 (BCIN_05g03680); Ss: $S$. sclerotiorum (XP_001593146.1, E-value: 8e-45, 80\% identity); Ab: Aspergillus bombycis (XP_022393145.1, E-value: 2e-39, 72\% identity); Af: A. flavus (XP_002382650.1, E-value: 2e-39, 72\% identity); Aa: A. alternata (XP_018388941.1, E-value: 1e-31, 58\% identity); An: A. niger (GAQ43359.1, E-value: 2e-31, 61\% identity); Cg: C. gloeosporioides (ELA33475.1, E-value: 4e-09, 44\% identity); Pz: Penicilliopsis zonata (XP_022579693.1, E-value: 5e-34, 63\% identity); Ar: Ascochyta rabiei (KZM20535.1, E-value: 2e-31, 58\% identity); Dh: Diaporthe helianthi (POS68622.1, E-value: 4e-31, 61\% identity); $\mathrm{Bm}:$ Bipolaris maydis (XP_014074148.1, E-value: 2e-29, 55\% identity). (B) Phylogenetic analysis of BcPTP1 and its homologs from other fungi. The full-length protein sequences were analyzed using MEGA $X$ with Unrooted neighbor-joining bootstrap (1,000 replicates). The black triangle mark location of BcPTP1. A scale bar at the lower left corresponds to a genetic distance of 0.1. (C) 3D structural models of BcPTP1, predicted using I-TASSER and further analyzed by PyMOL software. Left: cartoon models; right: surface models. 
984

A

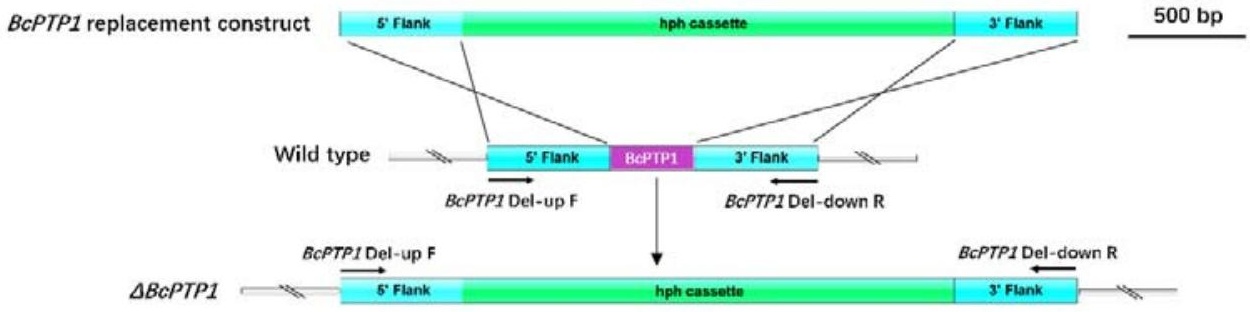

B

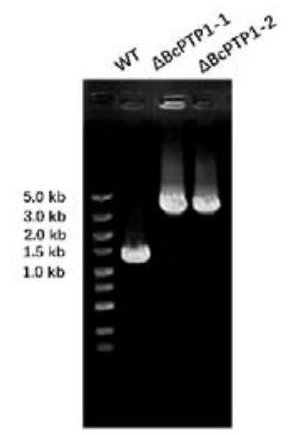

C

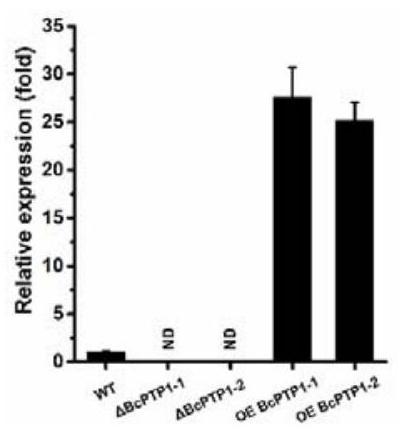

Fig. S3. The bcptp1 gene deletion strategy and confirmation in B. cinerea. (A) Strategy used to generate the bcptpl gene deletion mutants. The deletion construct used to transform the wild type strain contained the hygromycin resistance $(h p h)$ cassette flanked by upstream and downstream sequence of the bcptpl gene. The positions of the PCR primers used to verify the deletion transformants are indicated (BcPTP1 Del-up F and BcPTP1 Del-down R). The scale bar indicates 500 bp. (B) PCR amplifications carried out to verify the bcptpl deletion mutants using the 5' flank For and 3' flank Rev primers. As templates, genomic DNA from either the wild type strain or the bcptpl deletion mutants $\triangle B c P T P 1-1$ and $\triangle B c P T P 1-2$, was used as indicated. (C) RT-qPCR carried out to analyze the expression levels of the bcptp1 gene in the wild type strain, the bcptpl deletion mutants $\triangle B c P T P 1-1$ and $\triangle B c P T P 1-2$, the bcptpl overexpression strains OE BcPTP1-1 and OE BcPTP1-2. The strains cultured on PDA at $22^{\circ} \mathrm{C}$ for $72 \mathrm{~h}$ were used for RT-qPCR analysis. The relative levels of transcript were calculated using the comparative $\mathrm{Ct}$ method. The bcptpl gene expression level in wild type strain was set as level 1 . The transcript level of the $B$. cinerea bcgpdh gene was used to normalize different samples. Data represent means and standard deviations of three independent replicates. ND=not detected. 
A
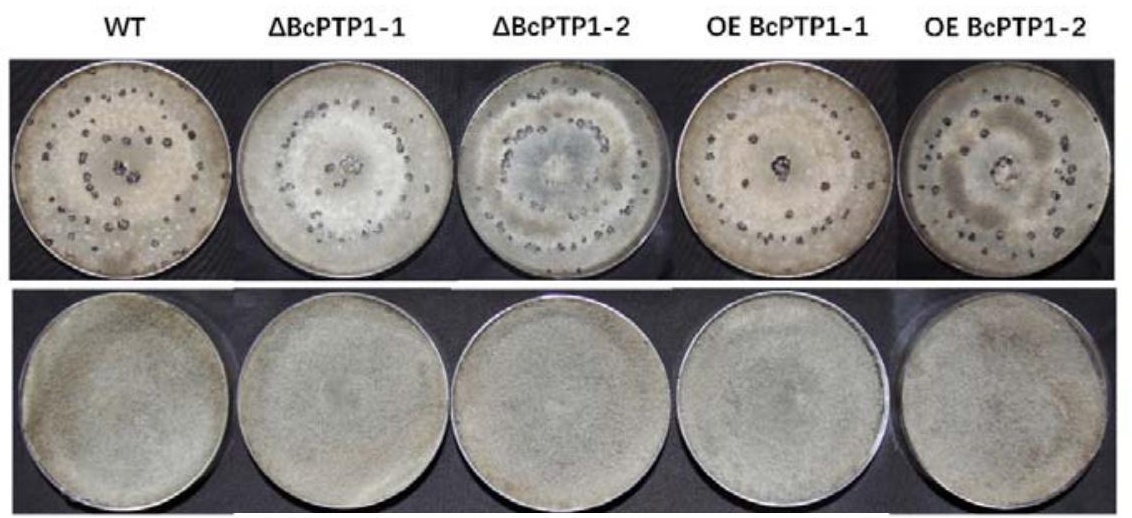

B

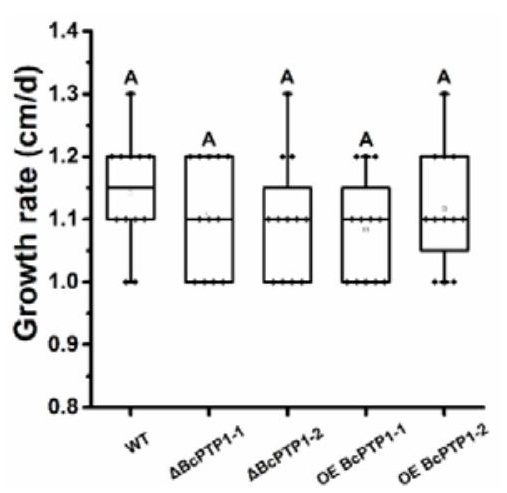

C

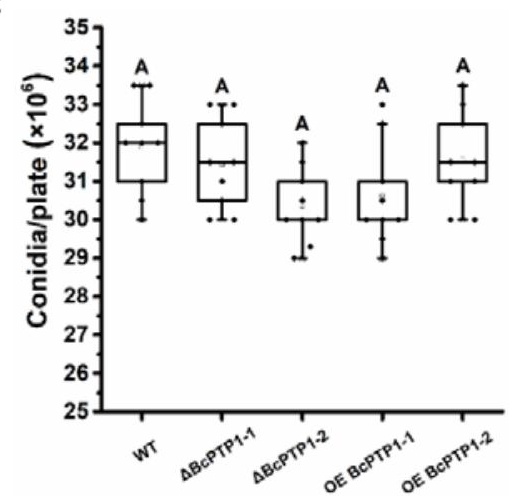

Fig. S4. Phenotypes of $B$. cinerea wild type, and the bcptp1 deletion and overexpression strains. (A) Colony morphology, sporulation and sclerotia formation. Top: colonies on PDA at $22^{\circ} \mathrm{C}$ for 15 days in complete darkness. Bottom: B. cinerea strains on PDA plates at $22^{\circ} \mathrm{C}$ for 7 days with continuous fluorescent light. (B) Hyphal growth rate. B. cinerea strains were grown on PDA plates at $22^{\circ} \mathrm{C}$ with continuous fluorescent light. Radial growth was measured every day for 5 days and growth rate was calculated. Data were obtained from three independent experiments, with four replicates in each experiment. In box plots, whiskers indicate the minimum and maximum values, the line indicates the median, the box boundaries indicate the upper (25th percentile) and lower (75th percentile) quartiles, all data are plotted as black dots. Same letters in the graph indicate no statistical differences at $\mathrm{P} \leq 0.01$ using one-way ANOVA one-way. (C) Conidial production of indicated strains cultured on PDA plates at $22{ }^{\circ} \mathrm{C}$ for 7 days. Conidiation of each strain was determined by collecting and counting conidia with a hematocytometer. Data were obtained from three independent experiments, with three replicates in each experiment. In box plots, whiskers indicate the minimum and maximum values, the line indicates the median, the box boundaries indicate the upper (25th percentile) and lower (75th percentile) quartiles, all data are plotted as black dots. Same letters in the graph indicate no statistical differences at $\mathrm{P} \leq 0.01$ using one-way ANOVA. 
1028

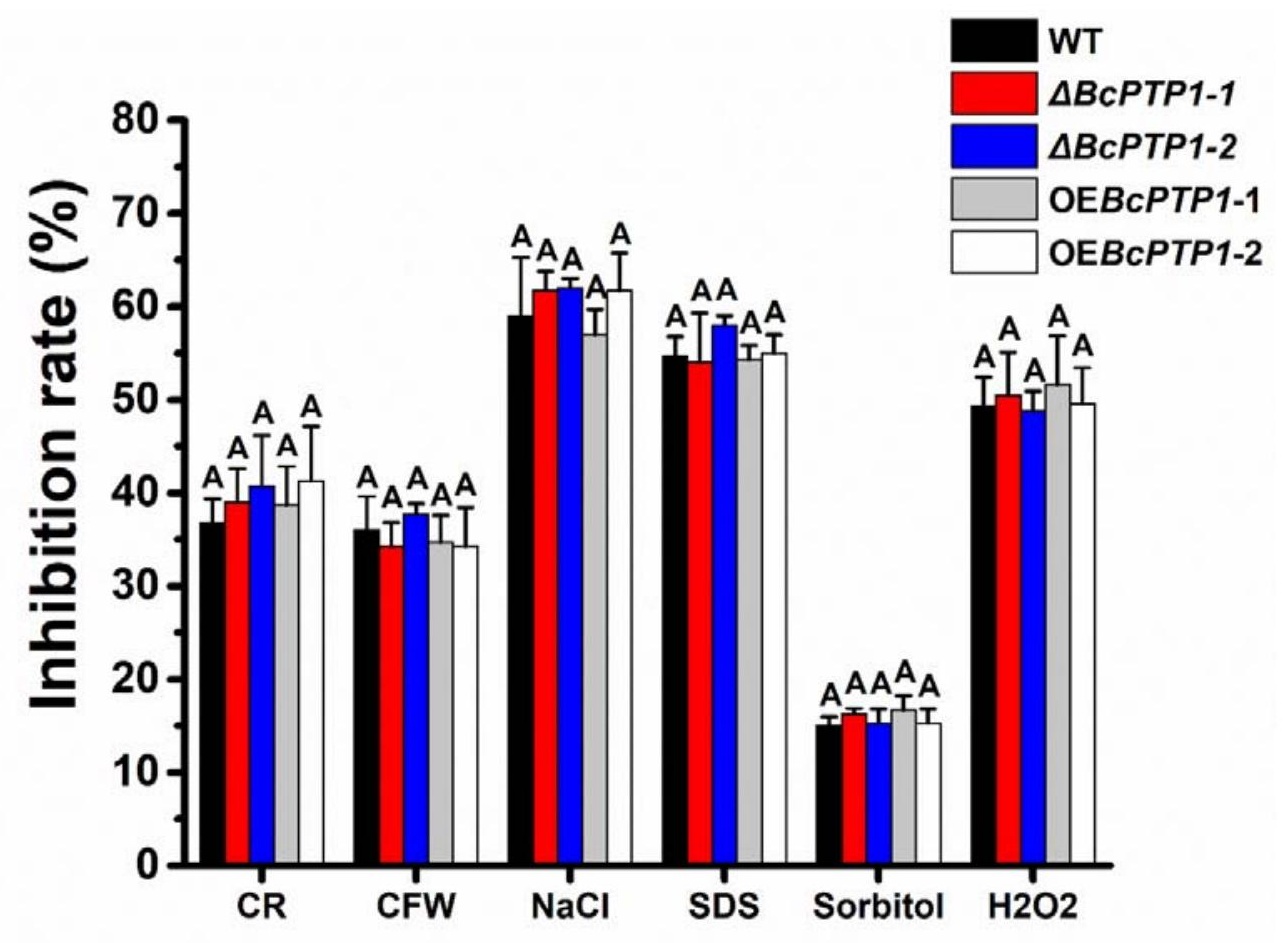

Fig. S5. bcptp1 deletion does not affect stress tolerance of $B$. cinerea. Inhibition rate of the radial growth of wild type strain, bcptpl deletion mutants and bcptpl overexpression strains were analyzed on PDA plates supplemented with $0.5 \mathrm{mg} / \mathrm{ml}$ Congo Red, $0.3 \mathrm{mg} / \mathrm{ml}$ Calcofluor White, $1 \mathrm{M}$ sorbitol, $1 \mathrm{M} \mathrm{NaCl}, 20 \mathrm{mM} \mathrm{H}_{2} \mathrm{O}_{2}$ and $0.02 \% \mathrm{SDS}$ at $22^{\circ} \mathrm{C}$, respectively. Data represent means and standard deviations from three independent experiments, each with three replications. Same letters in the graph indicate no statistical differences at $\mathrm{P} \leq 0.01$ using ANOVA (one-way). 


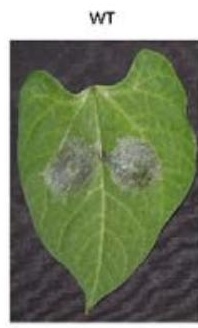

$\triangle \mathrm{BCPTP1-1}$

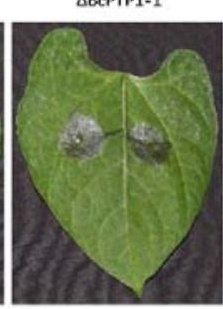

$\triangle B C P T P 1-2$

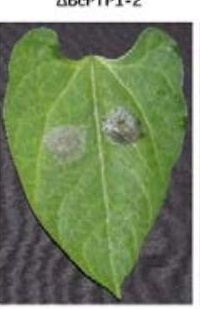

OE BCPTP1-1

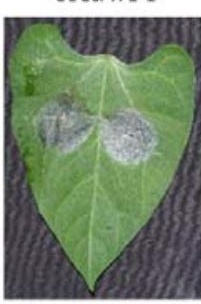

OE BCPTP1-2
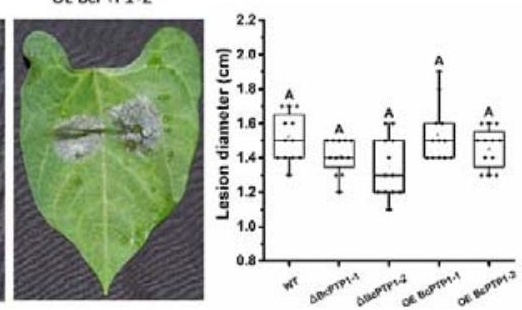

Fig. S6. Pathogenicity analysis of $\boldsymbol{B}$. cinerea strains. Beans leaves were inoculated with $7.5 \mu \mathrm{l}$ of conidia suspension $\left(2 \times 10^{5}\right.$ conidia $\left./ \mathrm{ml}\right)$. The plants were incubated in a humid chamber at $22^{\circ} \mathrm{C}$ for $72 \mathrm{~h}$, photographed and lesion size was determined. Data were obtained from three independent experiments, with four replicates in each experiment. In box plots, whiskers indicate the minimum and maximum values, the line indicates the median, the box boundaries indicate the upper (25th percentile) and lower (75th percentile) quartiles, all data are plotted as black dots. Same letters in the graph indicate no statistical differences at $\mathrm{P} \leq 0.01$ using ANOVA (one-way). 
1088 Table S1. Primers used for vector construction and PCR.

Primer purpose

bcptp1 deletion for upstream

bcptp1 deletion for downstream

bcptp1 over expression

bcptp1 transient expression

bcptp1 $^{\triangle S P}$ transient expression

GFP transient expression

bcptp1 for RT-qPCR

bcgpdh for RT-qPCR

GFP expression in E. coli
Primer name and sequence

BcPTP1 Del-up F: 5' TGTTGGGTCTTATGCGGAAAGAGAC 3'

$B c P T P 1$

Del-up

$\mathrm{R}$

5'CCTTTTTTTTTCAGGAATTATTCTCACAGTTTTGACGGTAGTGAAAAGG

ACTTTG 3'

BcPTP1

Del-down

F:

5'GATCTAGATGCATTCGCGAGGTACCGAGCTGAATCTCCGATGGGATGG

AGAGTTCT 3'

BcPTP1 Del-down R: 5’ ATCGATGGCTTGTTGTTCATCTTC 3'

BcPTP1-OE F: 5' GGCGCGCCATGGTCCGCATCTCCTCCAT 3'

BcPTP1-OE R: 5' GCGGCCGCTTACTGCTTGTAACATGGGGTACGATC 3'

BcPTP1-TE R: 5' TCTAGAATGGTCCGCATCTCCTCCAT 3'

BcPTP1-TE R: 5' CCCGGGCTGCTTGTAACATGGGGTACGATC 3'

BcPTP1 ${ }^{\triangle S P}$-TE R: 5' TCTAGAATGTGCACCTATTGCCAGTGCG 3'

$B c P T P 1{ }^{\triangle S P}$-TE R: 5' CCCGGGCTGCTTGTAACATGGGGTACGATC 3'

GFP-TE F: 5' TCTAGAATGGTGAGCAAGGGCGAGGAG 3'

GFP-TE R: 5' GAGCTCTTACTTGTACAGCTCGTCCATGCCG 3'

hph cassette F: 5' AGCTCGGTACCTCGCGAATGCATCTAGATC 3'

$h p h$ cassette R: 5' ACTGTGAGAATAATTCCTGAAAAAAAAAGG 3'

BcPTP1-q F: 5' TCACTGCTGTGTCTATTCGGATG 3'

BcPTP1-q R: 5' CGCACTTGTATTTTCCTCCAGC 3'

Bcgpdh-q F: 5'CGAAGAATAGCACAAACAGCTGGAC 3'

Bcgpdh-q R: 5'CGTCACCTTATGCTTCTTGCTCC 3'

GFP GST F: 5' CATATGGTGAGCAAGGGCGAGGAG 3'

GFP GST R: 5' CTCGAGTTACTTGTACAGCTCGTCCATGCCG 3' 
BcPTP1 expression in E. coli

NbSOBIRl for RT-qPCR

NbPRla for RT-qPCR

$N b P R 2$ for RT-qPCR

NbPR4 for RT-qPCR

NbLOX for RT-qPCR

NbERF1 for RT-qPCR

NbHIN1 for RT-qPCR

NbWRKY7 for RT-qPCR

NbPTI5 for RT-qPCR
BcPTP1 GST F: 5' CATATGTGCACCTATTGCCAGTGCG 3'

BcPTPl GST R: 5'CTCGAGTTACTGCTTGTAACATGGGGTACGATC 3'

NbBAK1-q F: 5'GAGGTGGGAGGAATGGCAAA 3'

NbBAK1-q R: 5'TTGGCCCCGACAATTCATCT 3'

NbSOBIR1-q F: 5'CCAGCAAGTCACAGAAGGGA 3'

NbSOBIR1-q R: 5'CCAACACCACACCAAAGCTG 3'

NbPRla-q F: 5' CCGCCTTCCCTCAACTCAAC 3'

NbPRla-q R: 5' GCACAACCAAGACGTACTGAG 3'

NbPR2-q F: 5' AGGTGTTTGCTATGGAATGC 3'

NbPR2-q R: 5' TCTGTACCCACCATCTTGC 3'

NbPR4-q F: 5' GGCCAAGATTCCTGTGGTAGAT 3'

NbPR4- $q$ R: 5' CACTGTTGTTTGAGTTCCTGTTCCT 3'

NbLOX-q F: 5' AAAACCTATGCCTCAAGAAC 3'

NbLOX-q R: 5' ACTGCTGCATAGGCTTTGG 3'

NbERF1-q F: 5' GCTCTTAACGTCGGATGGTC 3'

NbERF1-q R: 5' AGCCAAACCCTAGCTCCATT 3'

NbHIN1-q F: 5' CCAACTTGAACGGAGCCTATTA 3'

NbHIN1-q R: 5' AGGCATCCAAAGAGACAACTAC 3'

NbWRKY7-q F: 5' CACAAGGGTACAAACAACACAG 3'

NbWRKY7-q R: 5' GGTTGCATTTGGTTCATGTAAG 3'

NbPTI5-q F: 5' CCTCCAAGTTTGAGCTCGGATAGT 3'

NbPTI5- $q$ R: 5' CCAAGAAATTCTCCATGCACTCTGTC 3'

NbEF1 $\alpha$-q F: 5' TGGACACAGGGACTTCATCA 3'

NbEF1 $\alpha$-q R: 5' CAAGGGTGAAAGCAAGCAAT 3' 
A
BCPTP1-GFP
$B C P T P 1^{\triangle S P}$ - GFP
$S P B C P T P 1$ - GFP
GFP
Empty vector
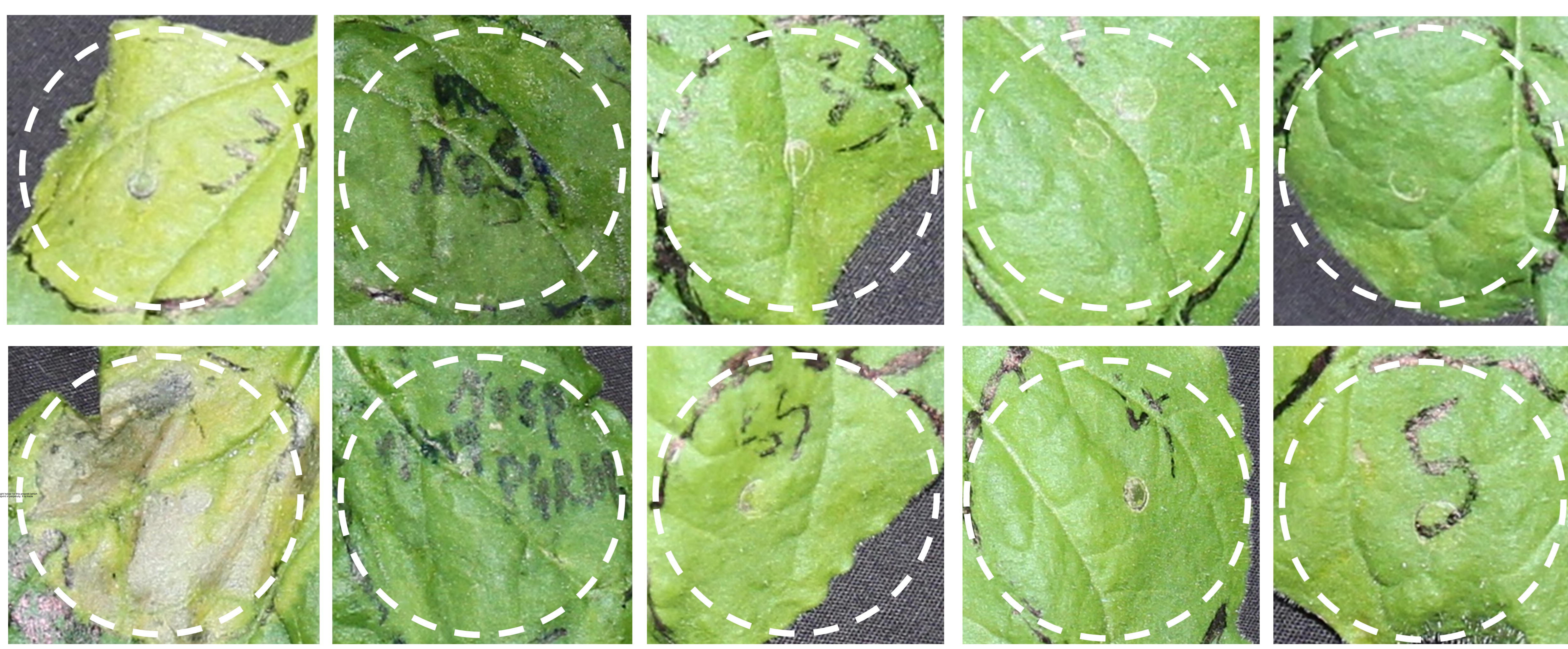

.
10 days

B
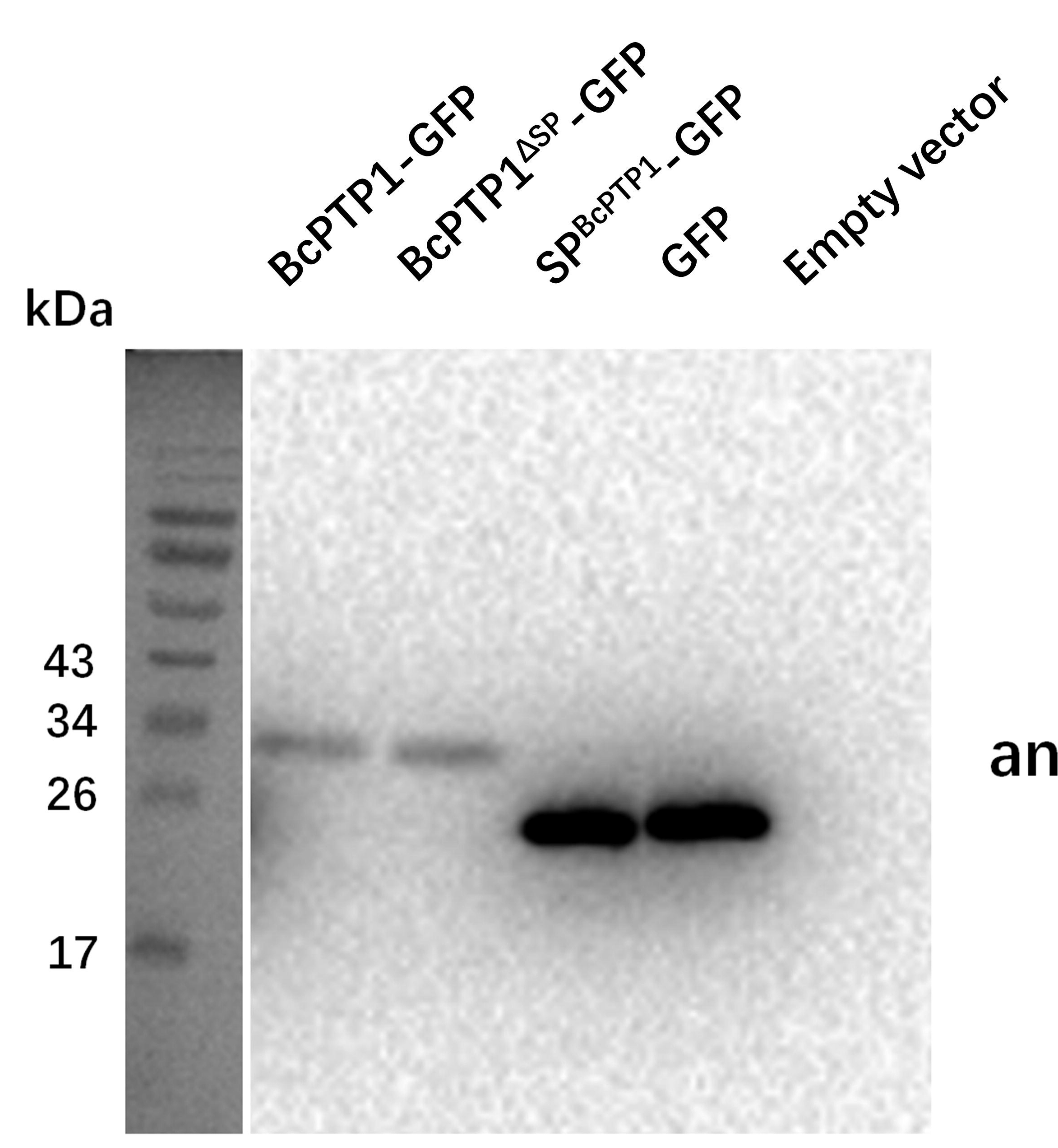

anti-GFP

15 days

Rubisco 


\section{A}
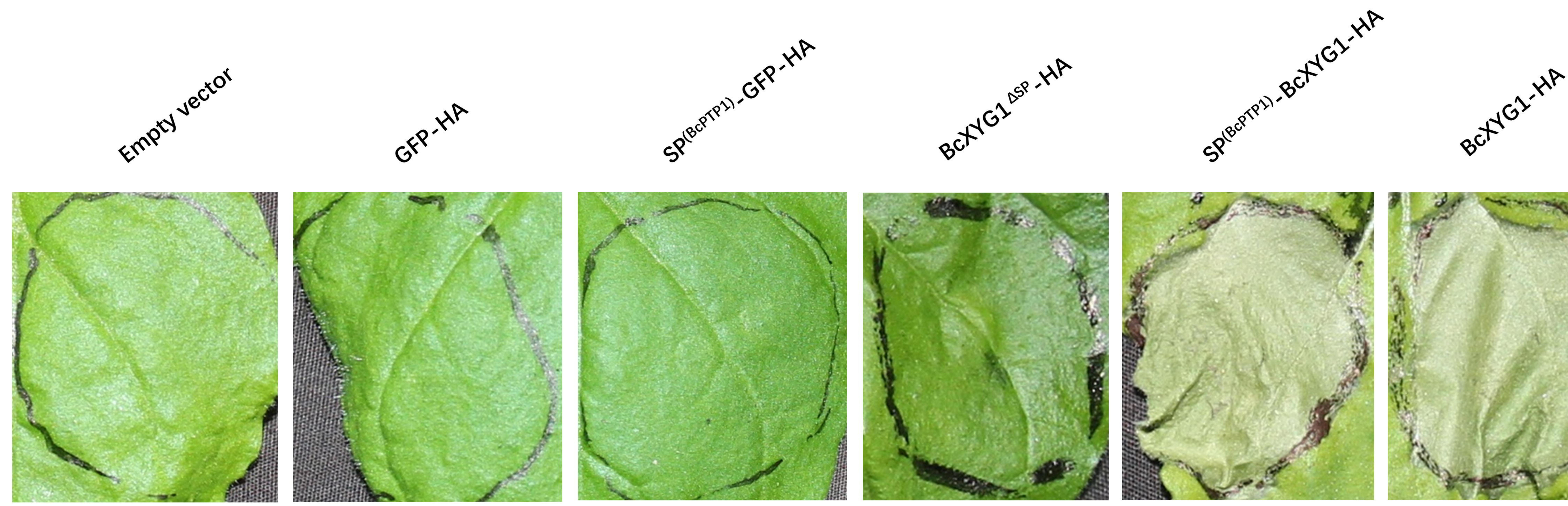

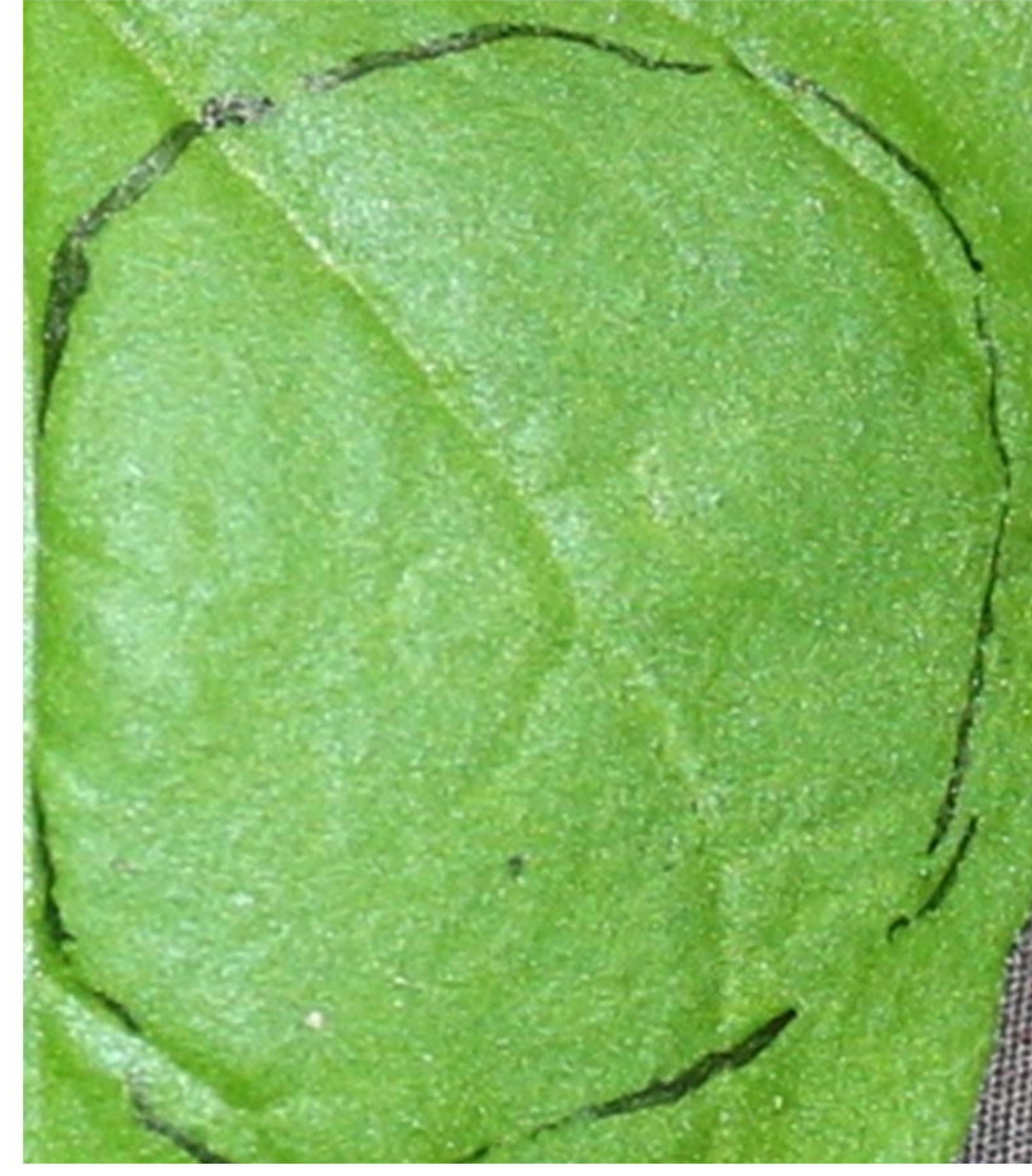

C
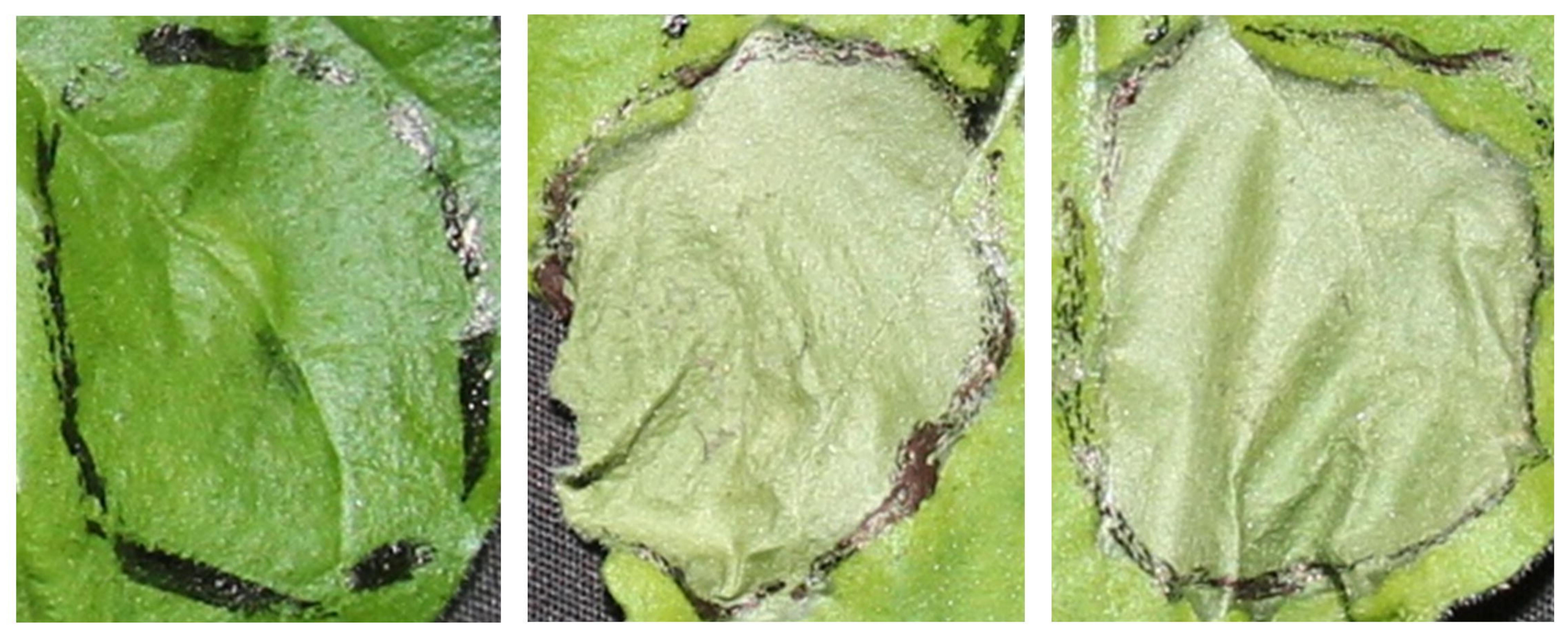

D

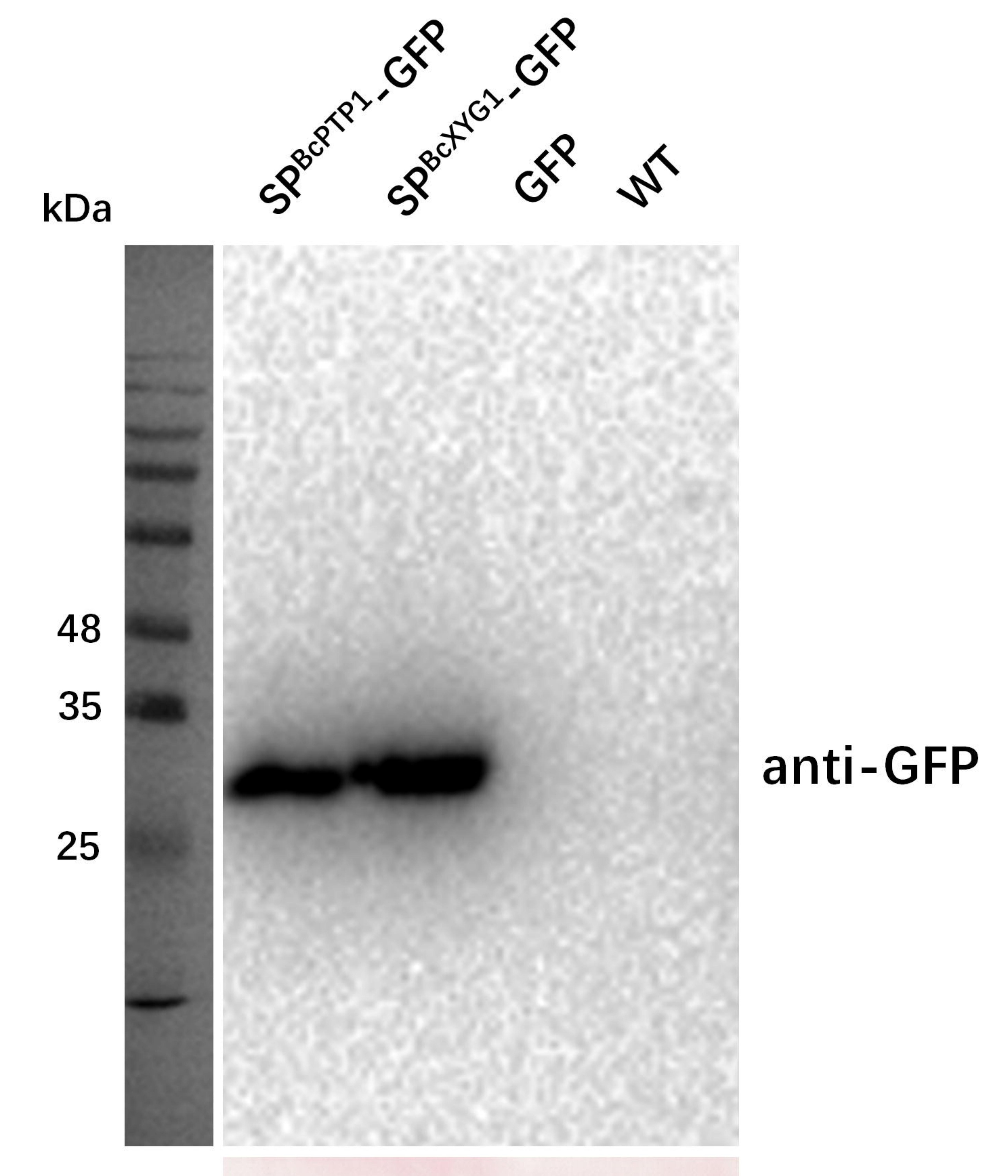

Ponceau S 





$A$

B
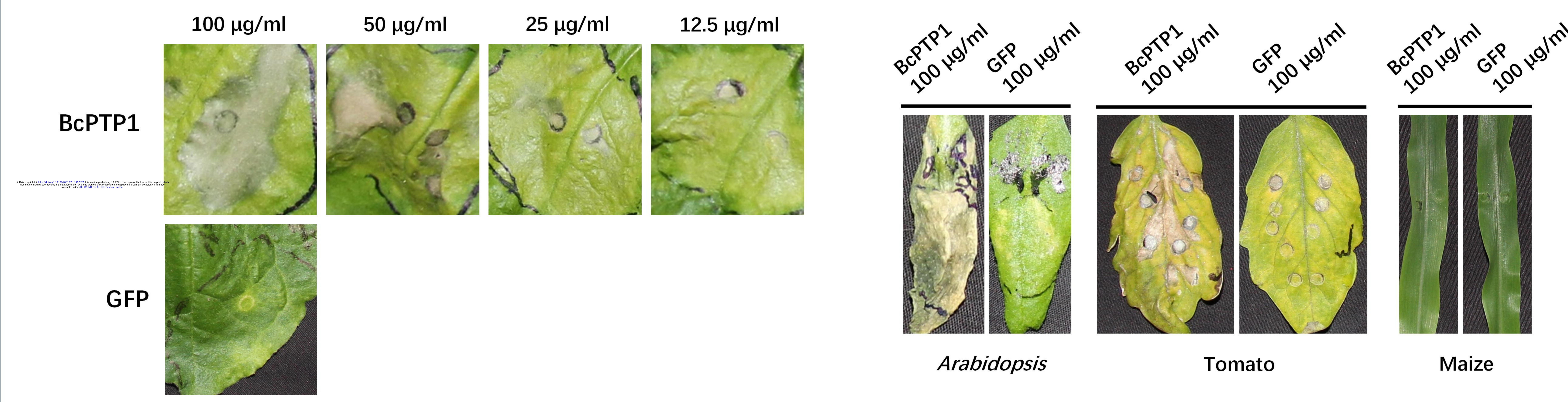



\section{$100^{\circ} \mathrm{C}$ treated $\quad 25^{\circ} \mathrm{C}$ treated}
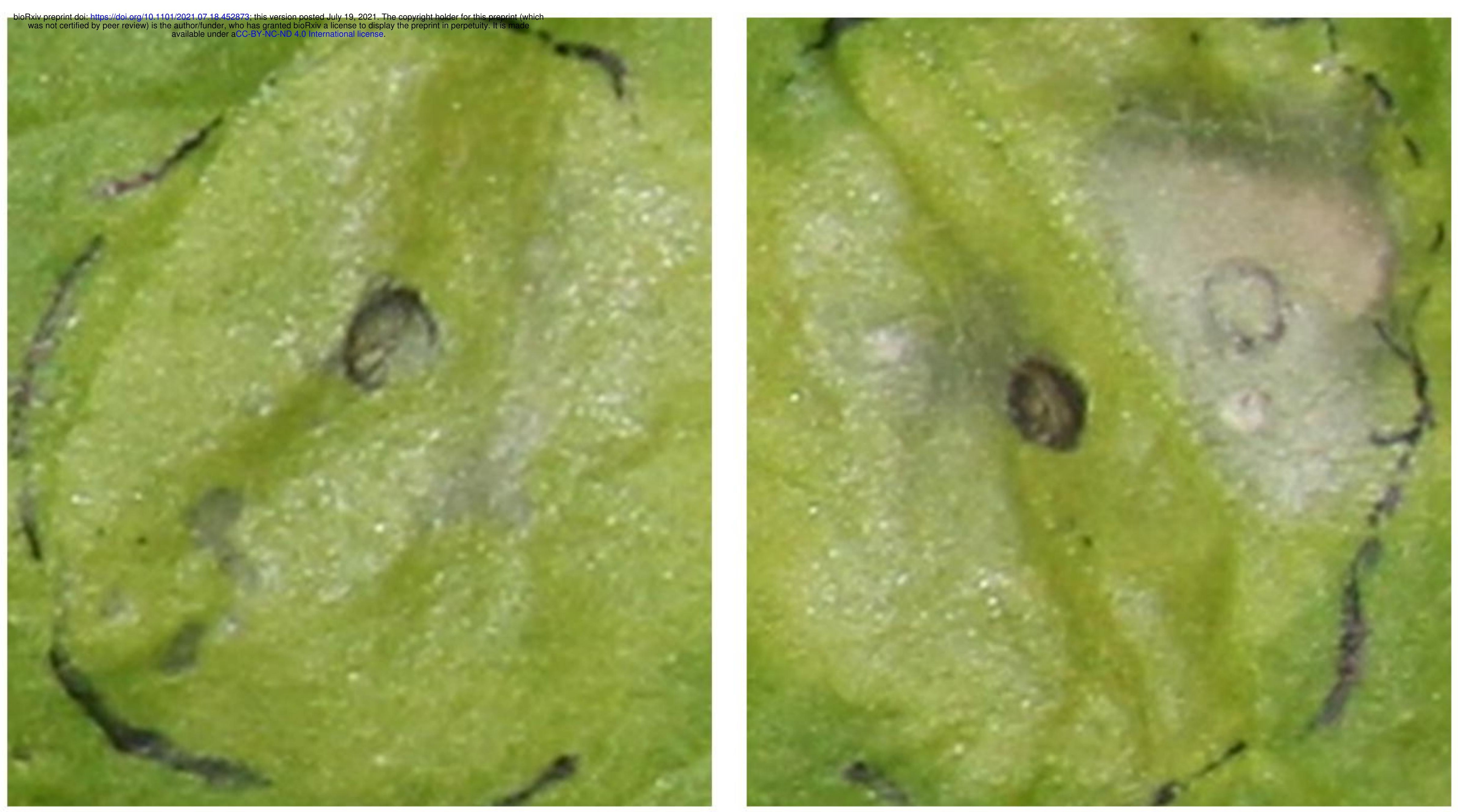


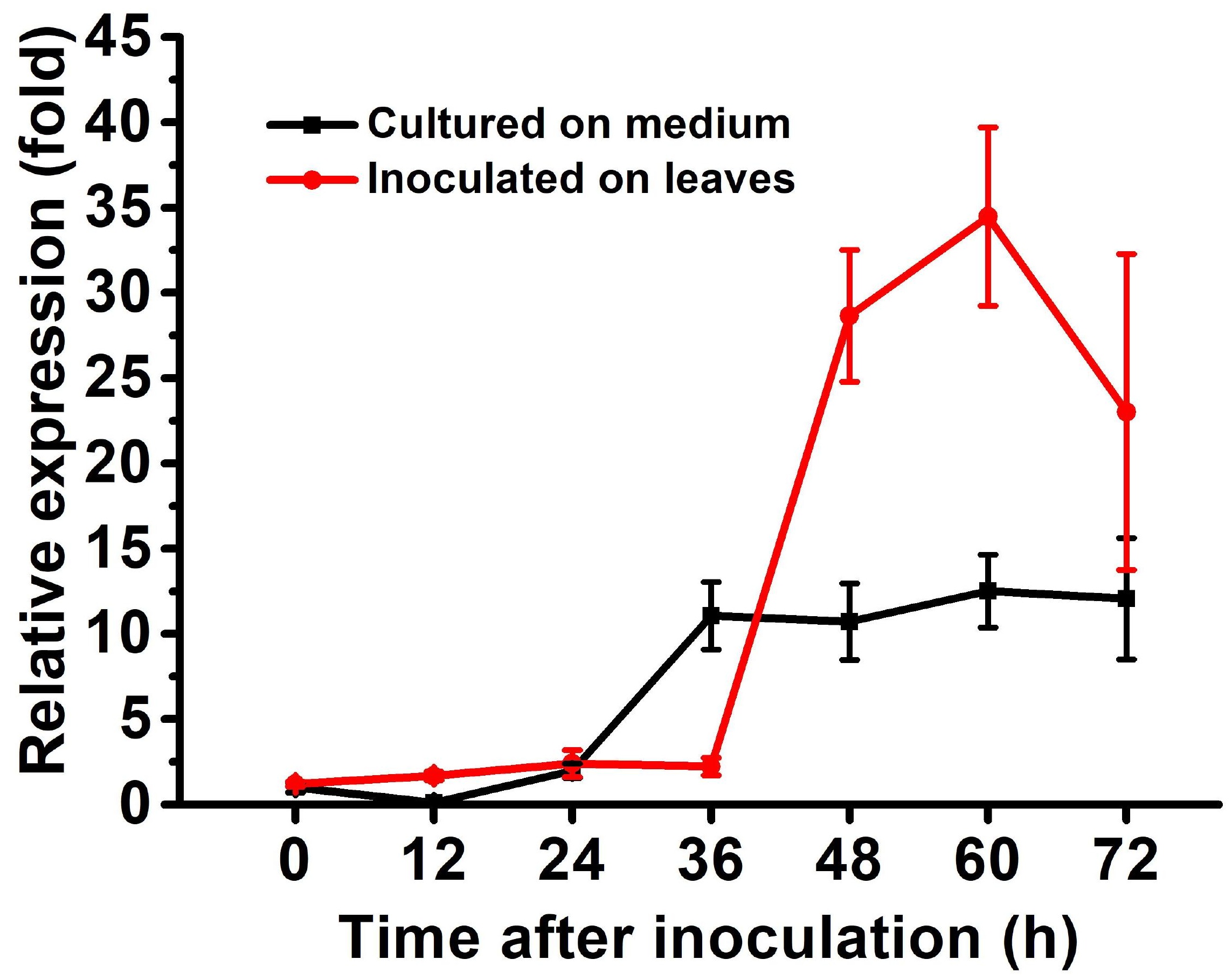


A
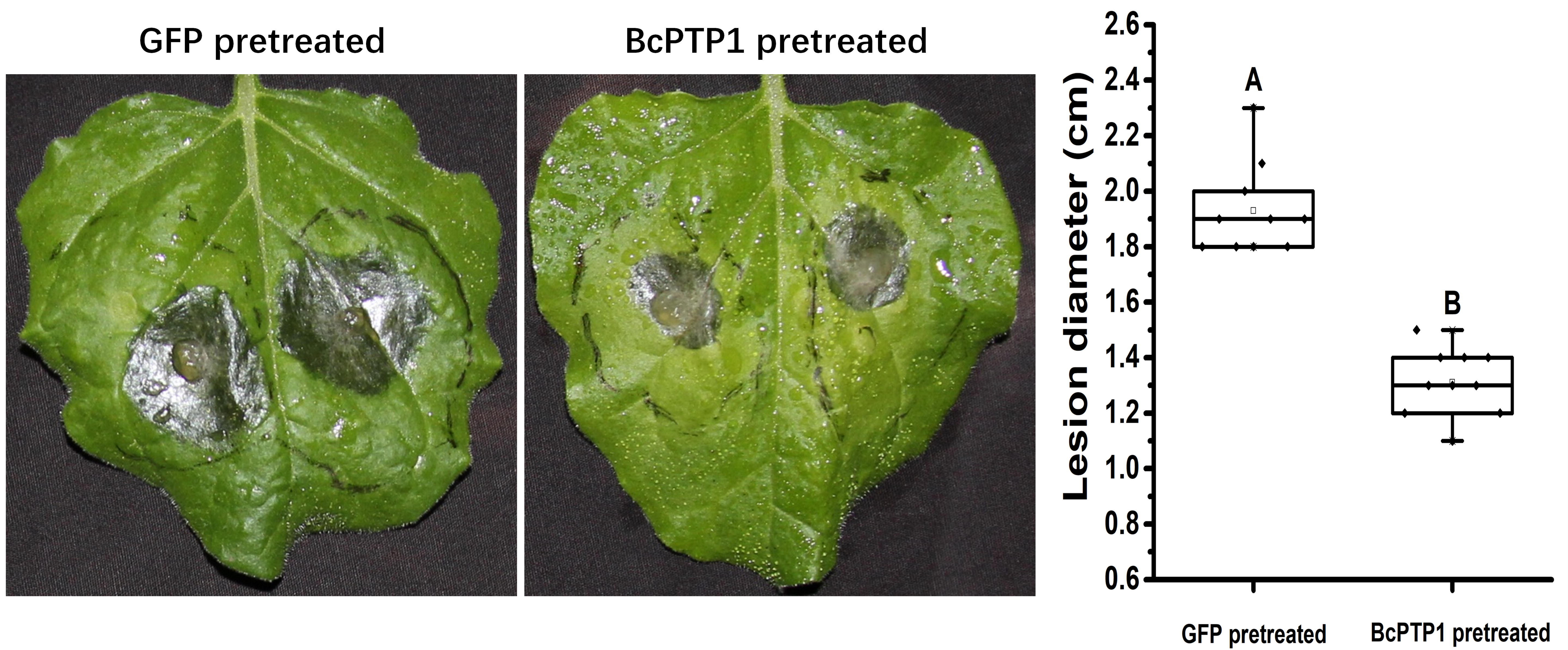

B

A

TRV:GFP
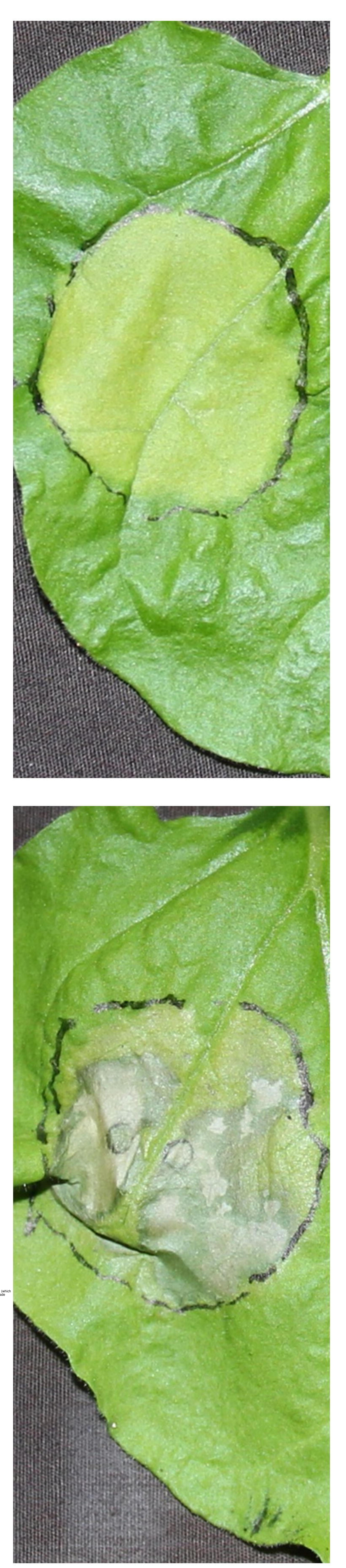

TRV:NbBAK1 TRV:NbSOBIR1
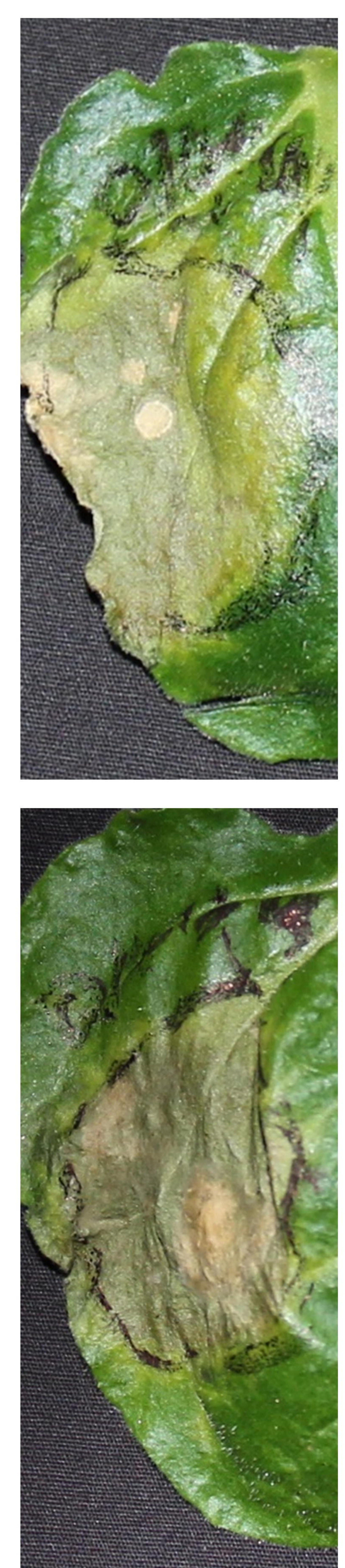

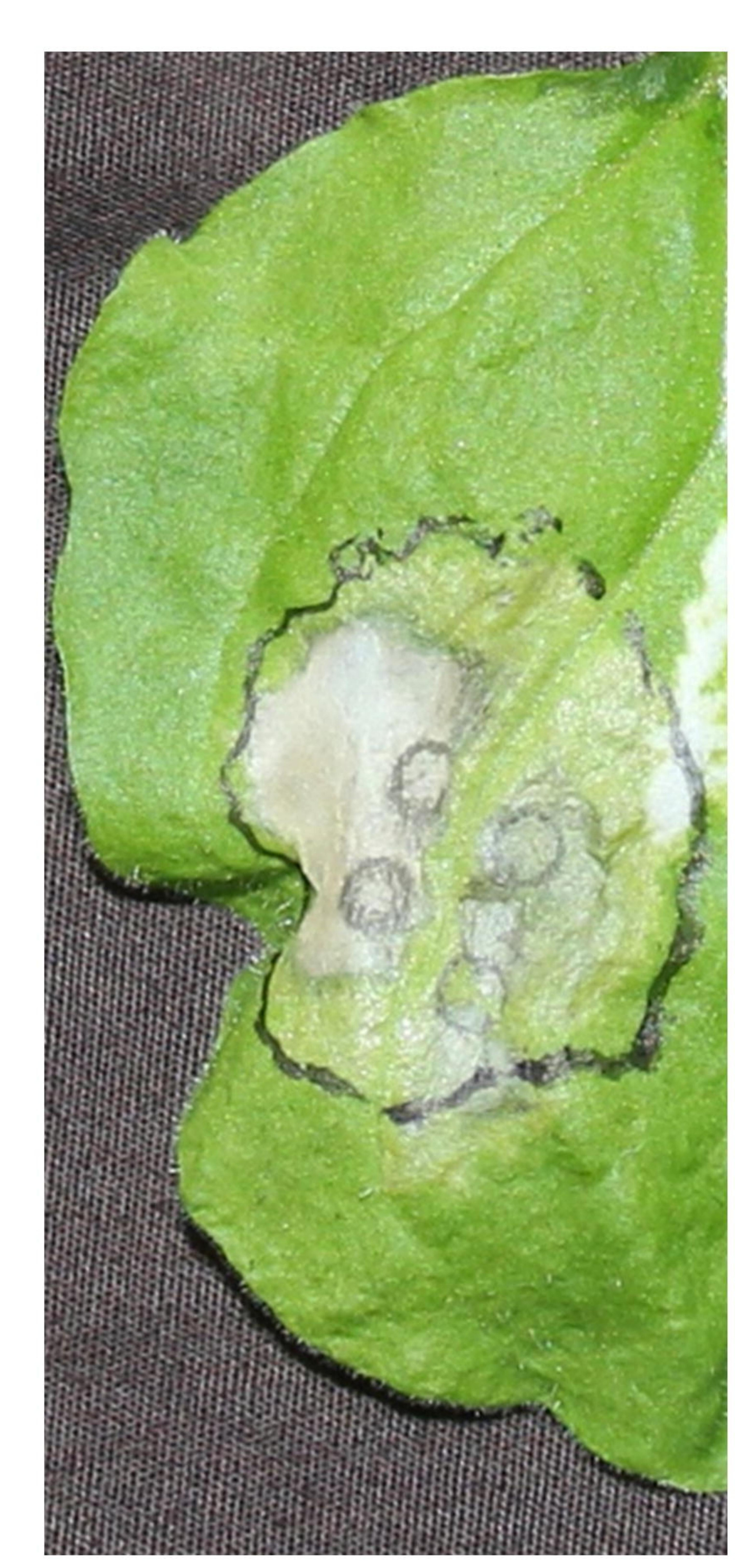

10 days

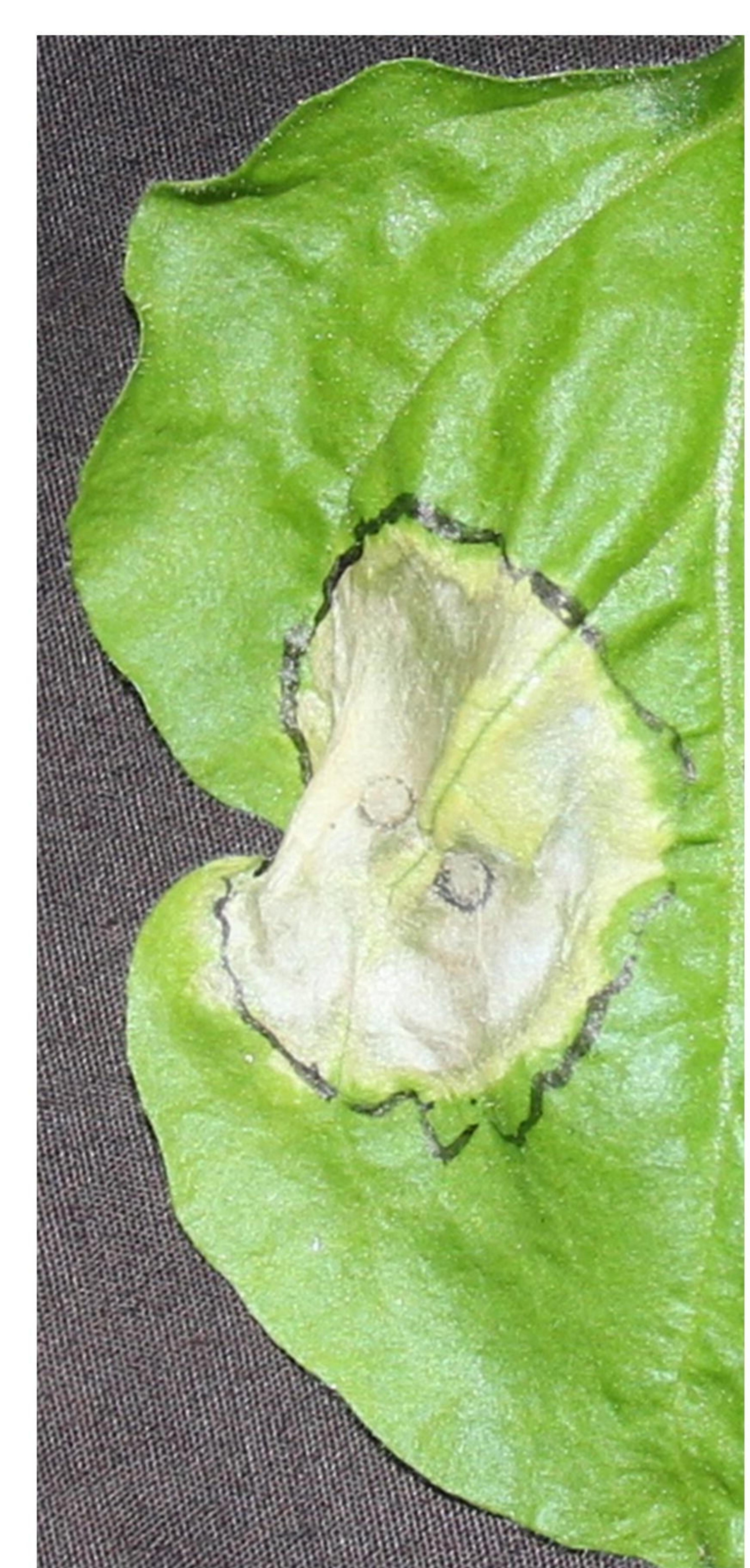

15 days
B

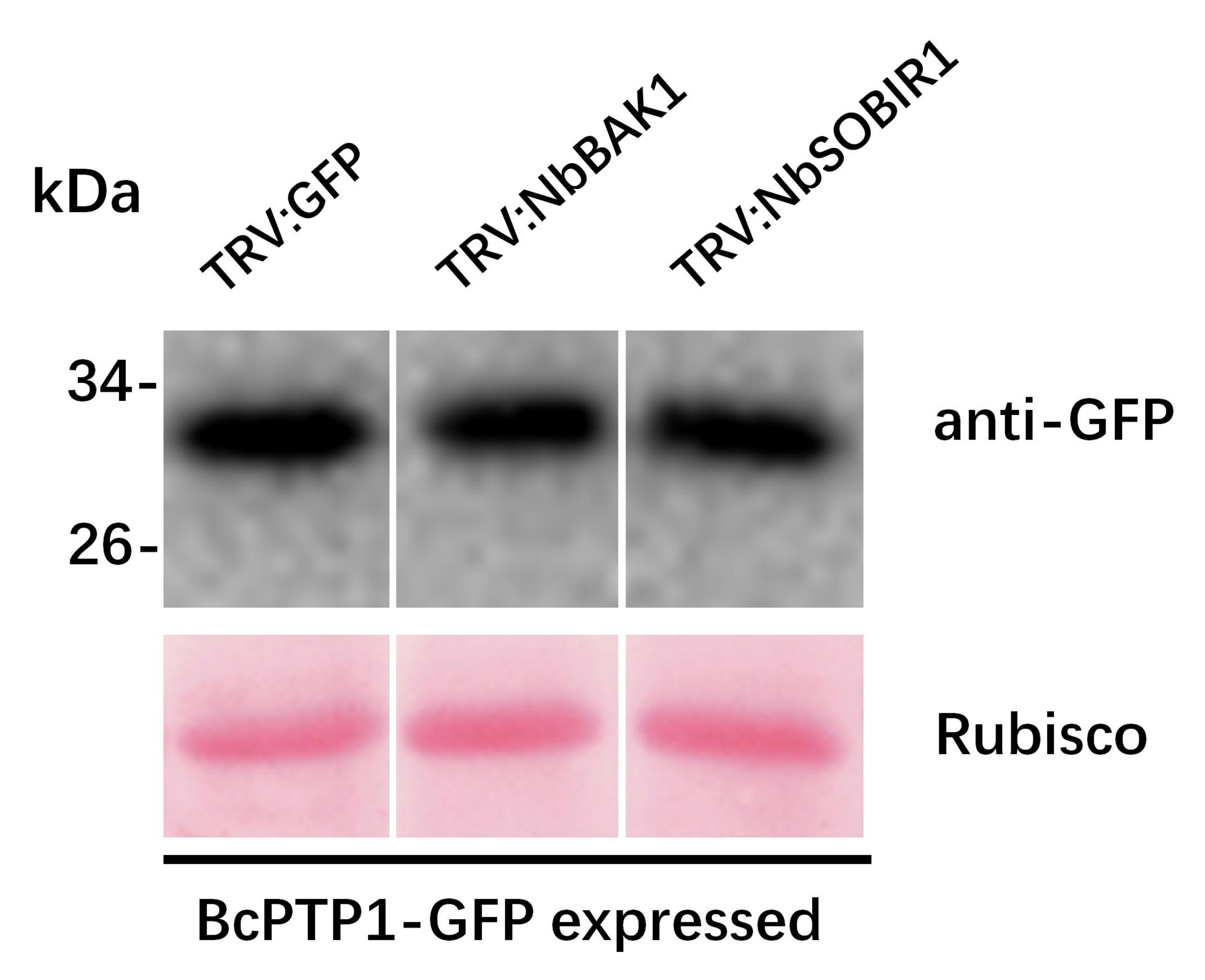

C
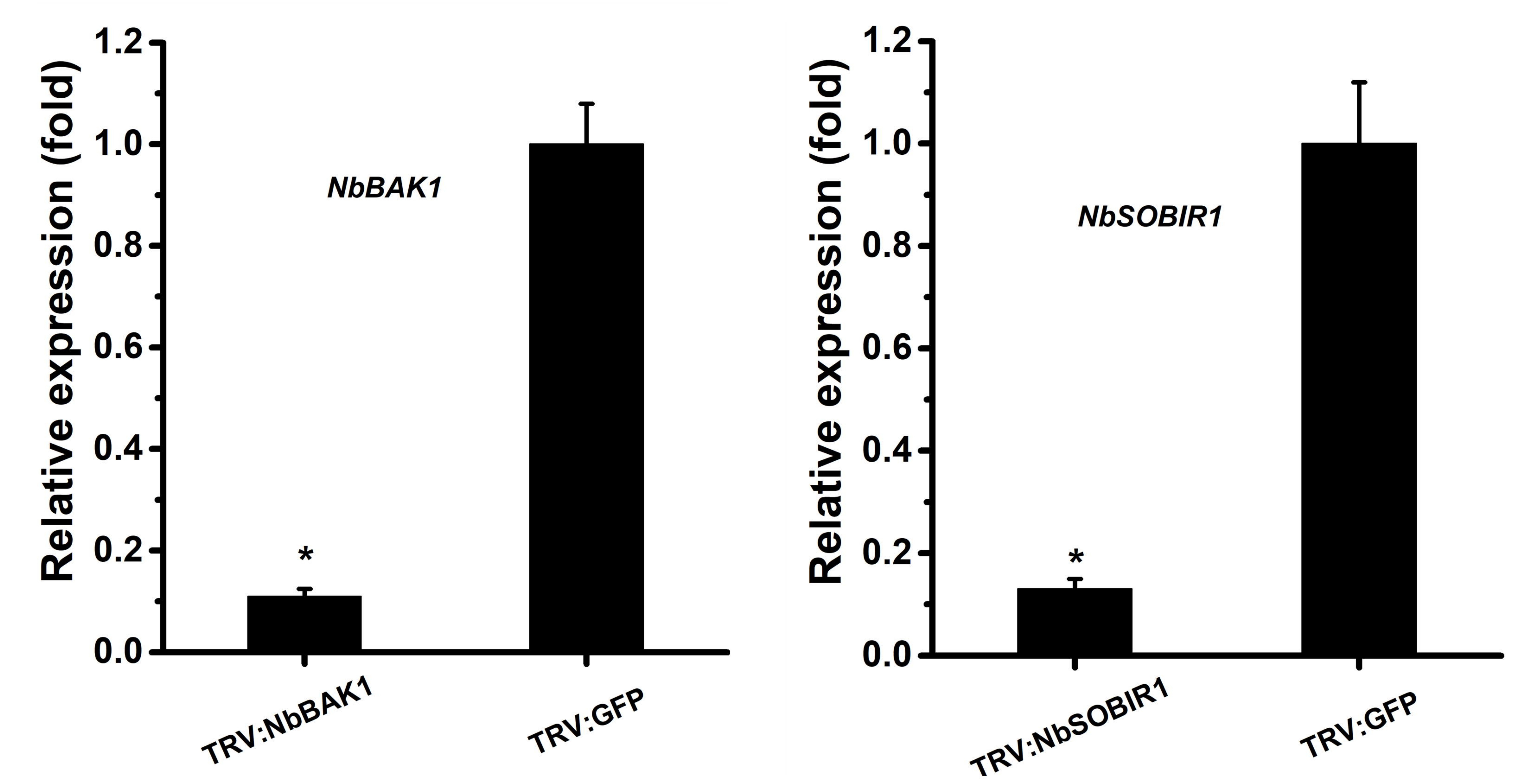


\section{Apoplast}

Botrytis cinerea

BcPTP1

Secretion

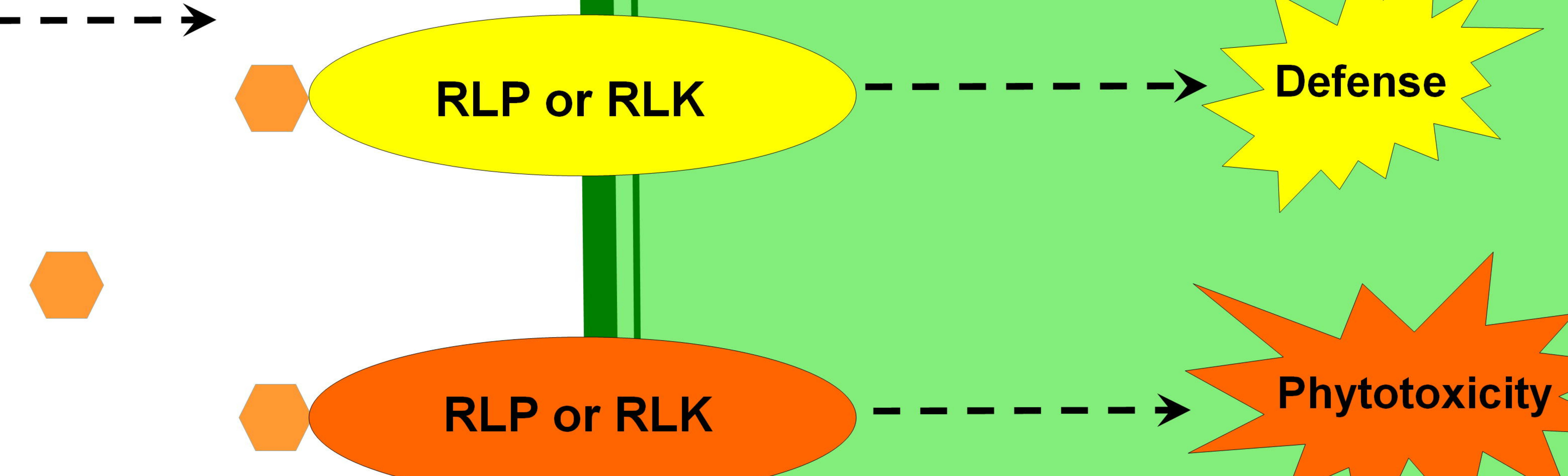

